\title{
Management of Brain Tumors in Eloquent Areas
}

\author{
José Manuel González - Darder and Pablo González - López \\ Department of Neurosurgery, Hospital Clínico Universitario Valencia \\ Spain
}

\section{Introduction}

Brain tumours in eloquent areas represent a great challenge for Neurosurgeons due to its surgical resection complexity. The main goal of this chapter is the comprehensive study of perioperative work-up, diagnostic and preoperative functional techniques, microsurgical skills and intraoperative monitoring for the management of brain tumours located in eloquent areas.

Integral management of brain tumors in eloquent areas involves different but related disciplines. So to reach a correct diagnosis, localization, treatment and prognosis, it is necessary to follow neuroclinical, neuroanatomical, neuroradiological, neurophysiological and neuropathological protocols. A complete resection is thought to be the best initial treatment for glial - type tumors, although there is no scientific evidence that a greater extent of resection is associated with a better prognosis (Berger et al., 1994; Keles et al., 2001, 2006; Pesudo et al., 2001; Sanai \& Berger, 2008; Shaw et al., 2008).

The infiltrative growth pattern of the neuropil by glial tumors is the reason for surgical treatment not being curative (Claes et al., 2007) and therefore the majority of patients will suffer a relapse or local progression of the disease some time after surgery. The known molecular mechanisms that drive this pattern of cellular migration have been deeply studied (Giese et al., 1995, 1996, 2003; Hwang et al., 2008). Although until new treatments for glial tumors are developed, the cytoreduction provided by surgery will maintain its value, because it offers material for diagnosis and research, alleviates both the focal and clinical symptoms of intracranial hypertension, and contributes to a greater efficacy of oncological treatments. Following this line of reasoning, there is a large number of modern series supporting the idea of resecting the largest possible volume of tumor (Giese et al., 2003; McGirt et al., 2008, 2009; Sanai \& Berger 2008). The price to pay for radical resection may be an increase in morbidity, fact that takes a special challenge in case of highly eloquent areas. In this sense, a great number of imaging, neurophysiological, neurochemical, and even surgical techniques have recently been developed and incorporated into the management of these tumors, with the ultimate objective being to optimize the resection limits; to extend them to the maximum while minimizing the eventual associated morbidity (Price et al., 2006; Provenzale et al., 2004; Stummer et al., 1998; Tanaka et al., 2009).

The final objective of this approach is to resect the lesion identified as tumoral based on the imaging techniques, as well as the neighboring brain tissue that is normal in appearance during surgery, but that is suspected of being invaded by tumor cells, or with cells already affected by the molecular disorder that would convert them into tumor cells. In this way, the 
tumoral resection limit would be taken to the point where there may be deterioration of any neurological function considered important (functional resection limit). This concept of functional resection limit extremes in case of brain tumors in eloquent areas due to the high eloquence of the surrounding brain tissue.

\section{Neuroanatomy of eloquent areas}

An accurate knowledge of function distribution over the human brain is necessary for the preoperatory planification development. The functional cortical and subcortical structures can be identified during tumor resection in different ways. In the same way, preoperative localization of intra-axial tumors and functional areas can be performed using multiple noninvasive imaging techniques.

\subsection{Motor function}

Most of brain areas related with motor function are located in the frontal lobe (Fig.1). The classical primary motor area is anatomically located in the precentral gyrus just anterior to the boundary between the frontal and parietal lobe, the central sulcus. In 1870, Gustav Fritsch and Eduard Hitzig published a paper entitled 'On the Electrical Excitability of the Cerebrum', which experimentally demonstrated that cortical stimulation of certain brain areas induced tonic muscle contractions in the contralateral body side. These areas have been largely studied since then, and the primary motor area has been demonstrated to be the area with a lower threshold for induction of movements after electrical stimulation. However there are other cortical areas related with the planning and control of movement. In this sense the Premotor and Supplementary Motor Area stand out. Both the premotor area and the supplementary motor area are included in the superior frontal gyrus, on its lateral and mesial side, right in front of the precentral sulcus. Other brain area related with the movement control remains located in the Primary Somatosensitive Area in the Parietal Lobe. This area is supposed to process and incorporate the nociceptive inputs to the motion generation. Some regions in the insular lobe have also been related with the motor control. All these brain cortical regions receive and send inputs and outputs to the corticospinal fiber tract in order to build coordinate movements. The corona radiata is a white matter region composed by these fibers, which come and go to different structures as the corpus callosum and especially to the internal capsule. The internal capsule can be subdivided in anterior and posterior limbs, and genu. The genu and posterior limb mainly contain motor and sensitive information. The motor fiber pathways get into the cerebral peduncles at the level of the diencephalo - mesencephalic junction, and finally decussate just before reaching the spinal cord.

\subsection{Sensitive function}

The path followed by the sensitive information is opposite to the motor system. It comes from the spinal cord through the medial lemniscus at the level of the mesencephalon. These fibers and the trigeminothalamic fiber tract join together and enter in the ventroposterior lateral nucleus of thalamus, where after synapsing are launched to the sensitive cortex through the superior thalamic peduncle, which joins to the posterior limb of the internal capsule at the level of the caudate body in the lateral wall of lateral ventricles. This sensitive information mainly travels to the Primary Somatosensitive Area located in the Postcentral Gyrus, posterior to the central sulcus in the Parietal Lobe. 


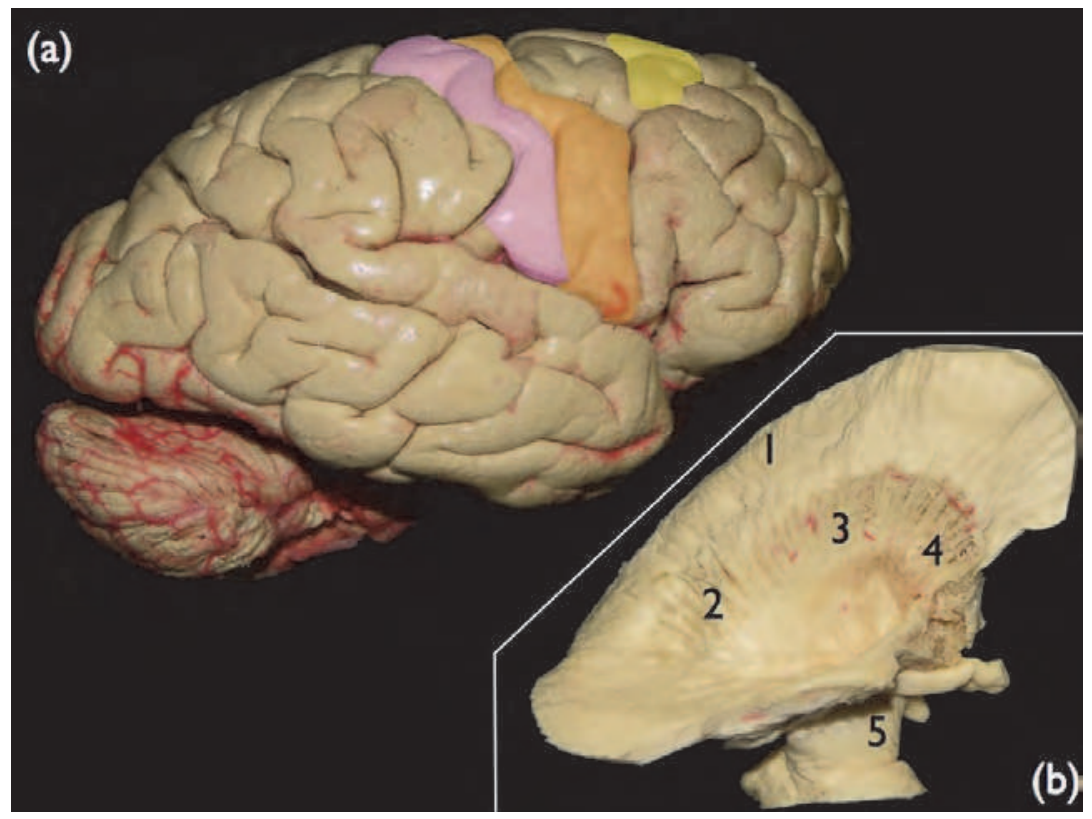

Fig. 1. (a) Right lateral view of the brain and cerebellar hemisphere in a cadaveric specimen after removing the arachnoid and vessels of the supratentorial cortex, where it is possible to appreciate the primary motor area in orange, the primary somatosensitive area in pink, and part of the supplementary motor area in yellow color; (b) right lateral view of the internal capsule where it is possible to appreciate the corona radiata (1), genu (3), anterior (4) and posterior (2) limbs of internal capsule, and caudally the cerebral peduncle (5)

\subsection{Speech}

In the XIX century, Paul Broca first described the language area after studying brain specimens of patients suffering aphasia. This area, nowadays known as Broca's Area, is anatomically located in the Inferior Frontal Gyrus of the dominant Frontal Lobe, filling the pars triangularis and opercularis (Fig.2). This area is essential in producing fluent speech. A lesion of Broca's area results in a non-fluent aphasia. Patients emit few words, both spoken and written, and understanding is maintained. The nondominant inferior frontal gyrus is involved in controlling the rhythm, intonation and emotion of verbal content, which is known as prosody. Therefore, any injury on that region will produce aprosodia, which is the lack of emotion in words. Both the premotor and supplementary motor area of the dominant hemisphere, are also involved in speech production. Speech also seems to consist of different areas located in the cortex of the temporoparietal and insular dominant hemispheres, as well as scattered groups of neurons that are activated parallely. The anterior commissure is a white fiber bundle that connects both temporal lobes and is supposed to be integrated in the speech circuit. However the main fiber tract involved in language comprehension and production is the superior longitudinal fasciculus, which connects the frontal, parietal, occipital and temporal lobes. This fasciculus is the longest of the association fiber bundles. It remains superior to the lentiform nucleus and lateral to the insula. 


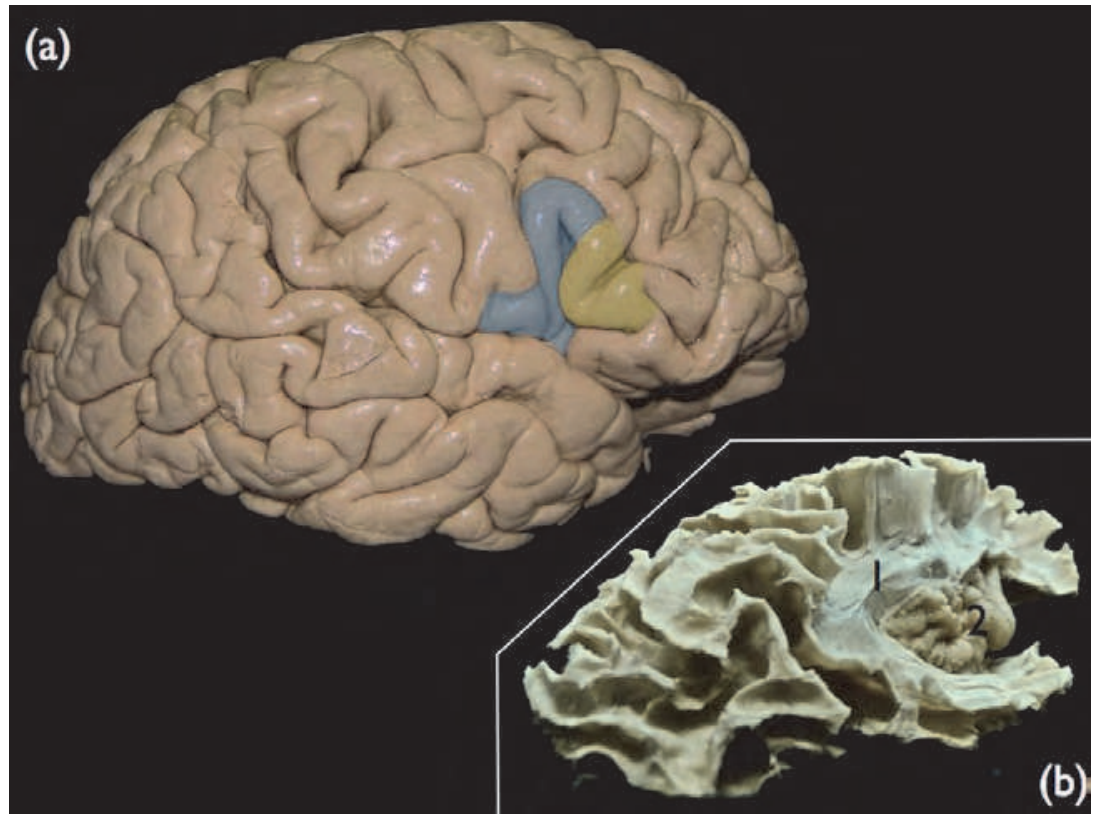

Fig. 2. (a) Right lateral see of the brain where it is possible to view in blue the pars opercularis and in yellow the pars triangularis; (b) posterolateral view of the superior longitudinal fasciculus (1) surrounding the insular lobe (2)

\subsection{Vision and eyes movement}

The Primary Visual Area, also known as striate cortex, is located in the Occipital Lobe, surrounding the calcarine sulcus. The visual information travels from the optic nerves and tracts till reaching the lateral geniculate bodies, from where after synapsing, the electrical impulse is transmitted to the striate cortex through the optic radiations, which remain located in the deep inferior temporal gyrus surrounding the temporal horn of lateral ventricles, is integrated in the posterior thalamic radiations and sagittal stratum at the level of the corona radiata, and finally ends in the occipital lobe surface.

Related with the process of vision it is important to highlight the Frontal Eye Field, which is ahead of the representation of the face and hand in the primary motor cortex, just behind the middle frontal gyrus and precentral sulcus. This area is responsible for saccadic eye movements, and their stimulation causes horizontal conjugate eye movements or oblique to the contralateral side. An injury of the frontal eye area can cause inability to turn its gaze on a voluntary basis to the contralateral visual field. Its main outputs or frontopontine projections travel together within the anterior limb of the internal capsule.

\subsection{Memory and learning}

A region highly implicated in learning and memory is located in the prefrontal cortex, receiving outputs from associative somatosensitive, visual and auditive areas. This area is responsible of the commonly known as working memory, related with planning and problem - solving and attention span. The orbital and medial prefrontal cortex receives 
connections from limbic structures like the amygdala. The mesial structures of the temporal lobe and the limbic circuit are also important structures in memory and learning ability. The limbic circuit is integrated by important mesial structures as the cingulum, amygdala, hippocampus, parahippocampal gyrus, fornix, mammillary body, mammillothalamic tract and some thalamic nuclei, among others.

\subsection{Reading}

J. Jules Dejerine in the XIX century first described different syndromes related to reading disability. The reading ability is known to be located mainly in the Gyrus Angularis in the Parietal Lobe, and posterior part of the Superior (Wernicke's Area) and Inferior Temporal Gyri in the Temporal Lobe, both in the dominant hemisphere.

\subsection{Hearing}

The auditory pathways are quite complex and include different white matter fiber tracts and nuclei in different levels of the encephalon. The main structures related with the audition are the vestibulocochlear nerve, cochlear nucleus, superior olive, inferior colliculus, medial geniculate body of thalamus, and finally the auditory cortex. The Primary Auditory Area is composed of the Transverse Gyrus of Heschl deep to the Superior Temporal Gyrus in the Sylvian Fissure, which crosses the upper surface and posterior Temporal Lobe.

\section{History and physical examination}

Physical examination remains of great interest in neurological diseases, but this fact takes on special significance when dealing with intrinsic brain tumors in Eloquent Areas.

Prior to modern neuroimaging, neurological treatment decisions were based on findings obtained from patient history and clinical examination. Despite the great information given by new imaging techniques, the clinical exam of associated neurological disturbances can be very helpful in order to identify intracranial tumors in the clinical practice (Maranhao et al., 2010). History and clinical exam offer important information to diagnose brain tumors and their location. Actually a detailed physical examination and a complete imaging analysis, is often enough as a perioperative assessment.

Clinical presentation of brain tumors commonly appears as irritative symptoms like seizures (almost $80 \%$ of intrinsic brain tumors; presenting and also once diagnosed). Although its high frequency, irritative symptoms do not clearly orient to the tumor location.

Neurological deficit is also a classic event referred in cases of brain tumors in eloquent areas, and the kind of neurological disturbance can help to clarify the tumor location. Tumors affecting the frontal lobe can show clinical findings like hemiparesis, conjugate deviation of eyes, fluent speech deficits or difficulty with word finding in case of dominant hemisphere and memory and learning difficulties. Patients diagnosed of brain intrinsic tumors affecting the Parietal Lobe can present hemiparesis, paresthesias and dysesthesias, and aphasia and symptoms as reading and hearing disturbances when affecting the dominant hemisphere. Parietal lobe tumors can even present hemianopia and spatial disorientation. Temporal Lobe tumors usually present a large variety and even mixed symptoms. The most common symptoms are temporal seizures. Lesions in the dominant hemisphere can show different degrees and varieties of speech, hearing and reading abnormalities. Memory and learning disabilities are also symptoms frequently found in this kind of tumors. Quadrantanopia is also a frequent symptom present after an ophthalmologic revision in temporal lobe tumors. 
Patients affected by Occipital Lobe intrinsic brain tumors can notice visual problems as contralateral hemianopia, although these findings can be misinterpreted, fact that makes necessary a specific visual examination. Insular lobe connects the limbic system and cortical areas, taking part in many superior functions, so tumors located in this deep area can affect sensitive, motor, speech and auditive tasks. Lesions affecting the limbic circuit usually present as cognitive disorders or memory disabilities. Subcortical lesions infiltrating or displacing important white matter fiber bundles can cause hemiparesis, sensitive and eyes movement disturbances in case of the internal capsule, memory and cognitive disorders in case of the uncinate fascicle, speech problems when affecting the superior longitudinal fascicle and of course visual problems in case of lesions damaging the optic radiations.

\begin{tabular}{|l|l|}
\hline Lobe affected & Symptomatology \\
\hline Frontal & $\begin{array}{l}\text { Personality and gait disturbances, expressive aphasia, hemipharesis, } \\
\text { conjugate deviation of eyes, seizures }\end{array}$ \\
\hline Parietal & $\begin{array}{l}\text { Sensory disturbances, receptive aphasia, hemianopia, spatial } \\
\text { disorientation }\end{array}$ \\
\hline Temporal & $\begin{array}{l}\text { Behaviour disturbances, hearing loss, comprehension inabilities, } \\
\text { symptoms of autism, memory loss, quadrantanopia }\end{array}$ \\
\hline Occipital & Hemianopia \\
\hline Insular & $\begin{array}{l}\text { Comprehension and expression disturbances, hemiparesis, } \\
\text { personality changes, memory problems }\end{array}$ \\
\hline
\end{tabular}

Table 1. Focal Neurologic Signs and Symptoms of Primary Brain Tumors

Brain tumors can also show different degrees of cognitive disabilities. These symptoms can be difficult to interpret because of the inability of most of these patients to perceive changes in memory and personality, and even to relate a comprehensive history. In this sense a close family member can help to uncove some clinical findings.

Moreover the type of symptoms, it is also really helpful exploring the timing. Evolutive and progressive changes orient to a tumor diagnosis. In this sense it remains of great interest to perform specific questions with the aim of remarking symptoms that could have been misinterpreted by the patient. Slow progression of symptomatology focuses to a low - grade tumor, while a rapid progression focuses the study to a high - grade tumor. The duration of symptoms varies greatly, due to the fact that some patients have harbored lower grade gliomas that have undergone malignant transformation, leading to longer histories than those high - grade gliomas that arise de novo.

\section{Medical management}

Initial medical treatment is almost always the first step in managing brain tumors. In addition to provide a definitive treatment, an important role of neurosurgeons taking care of patients recently diagnosed of brain tumors is to alleviate the most common appearing symptoms as increased intracranial pressure due to peritumoral edema, headache, medication side effects, pain, symptomatic seizures, venous thromboembolism, fatigue and cognitive dysfunction. Effective medical management results in decreased morbidity and mortality and improved quality of life for affected patients. Although there are relatively 
few studies addressing these issues (Wen et al., 2006), guidelines can be offered to maximize quality of life and minimize hospital admissions.

\subsection{Initial medical treatment}

\subsubsection{Peritumoral edema}

Tumor - associated vasogenic edema is due to increased capillary permeability in the blood - brain barrier. This vasogenic edema surrounding brain tumors contributes significantly to the morbidity experienced by patients, resulting in headache with emesis and even seizures exacerbation (Papadopoulos et al., 2001); when severe, this edema can result in herniation and death. Corticosteroids are routinely employed when treating patients affected of brain tumors with symptomatic mass effect and cerebral edema, resulting in an essential early part of brain tumor therapy (Kaal \& Vecht, 2004).

In selected cases, when a fast depletive treatment is required, other measures as elevation of the head of the bed, diuretics, or fluid restriction, can be chosen till the steroid therapy becomes effective. Although the mechanism is not well known yet, steroids are thought to reduce the permeability of tumor capillaries.

The empirical initial dose of steroids is an intravenous bolus of $10 \mathrm{mg}$ of dexamethasone, followed by a maintenance dose of $4 \mathrm{mg}$ every 6 hours in patients with significant symptomatic edema. Complete absorption from gastrointestinal tract is quite fast, and oral therapy should be used as soon as possible. Higher doses to these proposed can be employed in patients who do not respond to the usual initial dose. Response to steroids must be measured in terms of neurological deficits improvement during the first 48 hours. The dose may be increased up to $100 \mathrm{mg} /$ day when necessary (Vick \& Wilson, 1985). For asymptomatic patients with peritumoral edema on neuroimaging studies, corticosteroids are usually unnecessary.

Unfortunately corticosteroids are associated with a number of side effects, and the benefit should be balanced with the appearing of common side effects. The most common and important to notice complications in patients with brain tumors during steroid treatment are gastrointestinal disturbances, insomnia, manic behavior and hyperglycemia. Most of these side effects are solved after withdrawal of therapy (Wen et al., 2006). Patients with diabetes should be daily monitored in order to control their glycemic values to modify insulin dosage when necessary.

\subsubsection{Seizures}

Seizures are the presenting symptom of a brain tumor in up to $40 \%$ of patients and occur during the course of the illness in more than $60 \%$ of patients with tumors in the cerebral hemispheres (Moots et al., 1995). A cortical location of tumor is particularly common in patients with tumor - related epilepsy, with white matter and deep gray nuclei less common. Although the pathogenesis is poorly understood, some factors may be involved, as altered peritumoral amino acids, alteration and distribution of glutamate receptors, $\mathrm{pH}$, neuronal and glial enzyme and protein expression (Schaller \& Ruegg, 2003).

Antiepileptic drug treatment is universally accepted when a recognized seizure has appeared. However, there is strong evidence against the prophylactic use of antiepileptic drugs in patients just diagnosed brain tumors and free of seizures (Glantz et al., 2000).

The treatment should be performed with standard antiepileptic drugs. The selection of drugs usually depends on the surgeon's preferences. Phenytoin is the most commonly used 
antiepileptic when treating and preventing seizures in neurosurgery. Its pharmacokinetics remains quite variable among different patients, so its blood levels must be monitored until reaching the clinically effective serum level $(10-20 \mathrm{mcg} / \mathrm{mL})$. A loading dose must be employed at the beginning, inyecting $15-18 \mathrm{mg} / \mathrm{kg} / 24 \mathrm{~h}$ spread in three doses: half a dose initially, a quarter after 8 hours, and the last quarter after 16 hours. The maintenance dose ranges between $5-7 \mathrm{mg} / \mathrm{kg} / 24 \mathrm{~h}$ spread in three doses (once each 8 hours again). Once effective serum levels have been achieved, the dosage usually ranges between $300-500 \mathrm{mg} / \mathrm{d}$ in three doses. With recommended dosage, a period of 7-10 days may be required to achieve a steady state. Sodium valproate is also commonly employed in case of epileptogenic brain tumors. As other antiepileptic drugs, serum levels are difficult to be reached at the beginning. The usually employed intravenous loading dose remains in $15 \mathrm{mg} / \mathrm{kg}$ spread in 10-20 minutes, continuing after half an hour with a continuous perfusion of $1 \mathrm{mg} / \mathrm{kg} / \mathrm{h}$. The optimum dose must be balanced attending to the clinical efficacy. The plasmatic efficient levels range between $50-125 \mathrm{mcg} / \mathrm{mL}$. Oral administration must be used as soon as possible, dealing 20-30 mg/ kg between 2 and 3 doses a day. Carbamazepine is usually administered with a gradual increase of dosage. The optimal dosage is 800-1200 a day. Although its antiquity, phenobarbital is still used in cases of refractory symptomatic seizure disorders in patients affected by intrinsic brain tumors.

The unnecessary use of antiepileptic agents can alter the metabolism of many other drugs as steroids, chemotherapeutic agents and other antiepileptic, after inducing hepatic P450 enzymes (phenytoin, carbamazepine, phenobarbital). Actually there are a large number of agents free of inducing hepatic enzymes, with relatively few drug interactions (French et al., 2004).

Although infrequent, patients with brain tumors comprise 4-12\% of patients developing status epilepticus (Wen et al., 2006). Intravenous administration of antiepileptic agents is necessary in these cases.

\subsubsection{Reduced mobility}

Venous thromboembolism is a common cause of morbidity and mortality among patients with brain tumors of all types, particularly during the perioperative period (Rosenfeld \& Pruitt, 2007). This fact takes on special significance when dealing with brain tumors in eloquent areas, specially in motor areas, due to the reduced mobility from paretic limbs, which increase the risk for deep vein thrombosis and subsequent pulmonary emboli. An interesting study estimated the rate of symptomatic deep vein thrombosis to be $24 \%$ over the 17 months beyond the first 6 postoperative weeks. Although the pathogenesis is not completely understood yet, several risk factors have been identified: venous stasis in paretic limbs, age $>60$, larger tumor size, prolonged surgery, chemotherapy, high - dose steroid therapy and hormonal therapy (Marras et al., 2000).

Most neurosurgeons prefer the use of low - dose heparin (3500-5000 U subcutaneously/day), with which postoperative bleeding is uncommon.

\subsection{Preoperative medical treatment}

Preoperative medical protocols for surgery of brain tumors in eloquent areas differ from other brain tumors due to the special intraoperative techniques and anaesthesic management employed. 
General medical guidelines include steroid therapy, anticonvulsant agents and prophylaxis of venous thromboembolism in patients undergoing surgery for brain tumors. The effectiveness of prophylactic antiepileptic agents in patients who have undergone craniotomy is unclear. Different studies have showed no differences in risk of postoperative seizures between groups treated and no treated with anticonvulsant prophylaxis. However others have shown that prophylactic antiepileptic agents reduced the frequency of post operative seizures during the first week after a supratentorial surgery (North et al., 1983). In cases of awake or slept but non - relaxed surgery for brain tumors, the use of anticonvulsant drugs remains necessary and serum levels should be achieved days before surgery. The morning of surgery, antiepileptic dosage should be doubled $(10 \mathrm{mg} / \mathrm{kg}$ followed by 5 $\mathrm{mg} / \mathrm{kg} /$ day postoperative in case of phenytoin). However the dosage could depend on the tumor location, and definitive studies should be designed in order to adjust all the variables implicated. Preoperative steroids are used when peritumoral edema is obvious before surgery. As mentioned before, the effect of dexamethasone begins effective $24-48 \mathrm{~h}$ after its introduction. In case of preoperative treatment an initial bolus of $10 \mathrm{mg}$ followed by 10 $\mathrm{mg} / 6 \mathrm{~h} 24 \mathrm{~h}$ before surgery should be enough.

\subsection{Postperative medical treatment}

General medical management after a craniotomy performed to treat a brain tumor primary implies control of intracranial pressure due to cerebral edema, seizures due to cortex irritation, analgesia and deep vein thrombosis due to immobilization. Dexamethasone can be progressively reduced after surgery, but higher dosages are recommended during the first 24$48 \mathrm{~h}(6-8 \mathrm{mg} / 6 \mathrm{~h})$. Serum levels of antiepileptic drugs must be ranged between their clinical effective values. However, the most frequent systemic complication after craniotomy is deep vein thrombosis, reaching values of $1-10 \%$ of patients during the first month (Brandes et al., 1997). Multimodality venous thrombosis prophylaxis should begin at the time of the original surgery, taking patients out of bed and mobilized as soon as possible after surgery, using external leg compression until the patient is ambulatory and introducing anticoagulation with $5000 \mathrm{U}$ of heparin or low molecular weight heparin subcutaneously 24 hours after the craniotomy. However, it must not be forgotten the risk of bleeding (7/1000 intracranial hemorrhage and 28/1000 minor bleedings) (Hamilton et al., 2011).

Patients with primary brain tumors suffer a high risk of being affected by postoperative medical and surgical complications. The most accepted classification about postoperative complications after a craniotomy was performed in 1998 dividing them into three major groups: neurological, regional and systemic complications (Sawaya et al., 1998). Main neurologic postoperative deficits are motor, sensory, language and visual disturbances. When dealing with regional complications, we mean wound infections, CSF fistula, hydrocephalus, seizure, brain abscess, cerebritis and meningitis. Infectious processes require antibiotic drugs that cross the blood - brain barrier, and sometimes delayed surgeries. Other regional complications as hydrocephalus and CSF fistula usually require individualized mixed treatments. Seizures must be treated through antiepileptic drugs when there are not image findings, which justify them. Systemic complications include deep vein thrombosis, pulmonary embolus, myocardial infarction, urinary tract infection, pneumonia and sepsis. Each one of these entities requires individual treatments, but most of them, as neurological and regional complications, can be prevented through a careful preoperative planning, meticulous surgical technique, and attentive postoperative care. 


\begin{tabular}{|c|c|}
\hline Complication & Treatment \\
\hline \multicolumn{2}{|l|}{ NEUROLOGICAL } \\
\hline Motor/Sensory disability & \multirow{3}{*}{$\begin{array}{l}\text { Depending on the cause } \\
\text { (retraction, ischemic, } \\
\text { compression, bleeding, mass } \\
\text { effect) }\end{array}$} \\
\hline Aphasia & \\
\hline Visual disturbances & \\
\hline \multicolumn{2}{|l|}{ REGIONAL } \\
\hline \multirow{2}{*}{\begin{tabular}{|l|l|} 
Seizures \\
Hematoma
\end{tabular}} & Antiepileptic drugs \\
\hline & $\begin{array}{l}\text { Depending on the location and } \\
\text { clinical symptomatology }\end{array}$ \\
\hline Hydrocephalus & $\begin{array}{l}\text { External ventricular drainage } \pm \\
\text { Definitive shunt }\end{array}$ \\
\hline Pneumocephalus & $\begin{array}{l}\text { Observation vs. Revision } \\
\text { depending on the clinical } \\
\text { symptomatology }\end{array}$ \\
\hline Cerebrospinal fluid leak & $\begin{array}{l}\text { Medical treatment and/or } \\
\text { wound revisión }\end{array}$ \\
\hline Wound infection & $\begin{array}{l}\text { Surgical revisión and } \\
\text { Antibiotics }\end{array}$ \\
\hline Meningitis & Antibiotics \\
\hline \multicolumn{2}{|l|}{ SYSTEMIC } \\
\hline $\begin{array}{l}\text { Pulmonary, urinary tract, central line } \\
\text { infections and sepsis }\end{array}$ & Antibiotics \\
\hline Deep vein thrombosis & Anticoagulation therapy \\
\hline
\end{tabular}

Table 2. Postoperative complications in patients after craniotomies (Sawaya et al., 1998)

\section{Imaging}

Dealing with brain tumors has been dramatically changed since the improvement of diagnostic imaging techniques. Neuroimaging with either magnetic resonance imaging or computed tomography improves the accuracy of diagnosis and remains indispensable for surgical planning.

Nowadays, modern imaging techniques are necessary to detect or confirm a structural abnormality, localize and assess the abnormality, characterize the abnormality, plan the surgical approach and extension, predict the possible postoperative neurological deficits, orient to the metabolism and metabolites composition of the tumor and surroundings, understand the 3D anatomical relation of the tumor with brain eloquent areas (cortical and subcortical) through functional and anatomical sequences, as well as to rate the surgical extent of resection intra and postoperatively. 


\subsection{Diagnostic techniques}

\subsubsection{Computed tomography}

Computed tomography is a great screening method and almost always the most commonly used image modality for the initial patient evaluation, mainly in cases of supratentorial abnormalities, due to its accuracy and availability.

CT scanners provide enough specific information for the initial management of brain tumors. Once detected a lesion it is also important to try to characterize it, due to the immediate management differences between neoplasms and non - neoplastic processes. This is the main reason to perform a CT with the aid of contrast agents. This modality differentiates between intrinsic and extrinsic tumors, and is also helpful to delimitate the edema from necrotic or cystic areas.

Brain tumors may be hypodense, isodense or hyperdense on a noncontrast CT scan, depending on tumor location and histology. Isodense or small tumors with few peritumoral edema and mass effect can be difficult to be visualized on noncontrast CT, but highlight after contrast injection. Extra - axial brain tumors show a good resolution with CT and usually enhance after contrast administration, while low - grade gliomas and epidermoid tumors do not enhance. Although MRI is preferable for tumor definitive evaluation, CT is preferred to detect calcifications, intratumoral hemorrhage or bone abnormalities as destruction, erosion, infiltration and hyperostosis.

\subsubsection{Magnetic resonance imaging}

Despite initial CT scanners, MRI is nowadays the imaging method of choice in the pre therapeutical evaluation of brain tumors.

Usual MRI sequences let visualizing both normal anatomy and pathological findings. They are used in the clinical set for lesion detection, localization, characterization, determination of tumor extension, planning and guiding tumor biopsy and therapeutical actions, and monitoring of treatment response. Advanced MRI sequences allow semi-quantitative and quantitative measurements of several entities, such as movement of water molecules, cerebral blood volume and flow or chemical tissue composition (Cha, 2006).

\subsubsection{Anatomic imaging}

Brain tumors localization and relations with their surroundings reach the highest interest in cases of tumors in eloquent areas. Once diagnosed a brain tumor, the next point on its evaluation remains in the fact of its anatomic location. Some MRI techniques are recommended for this purpose: T1- and T2-weighted sequences, including FLAIR, after gadolinium T1-weighted sequences with at least one spin-echo sequence, and an additional T1-weighted 3D sequence. In order to achieve a good localization of tumors in the different eloquent areas, it is recommended to perform at least the T1- and T2-weighted sequences in the axial, coronal and sagittal planes of the space.

The goal of anatomic imaging sequences in case of brain tumors in eloquent areas is centered on identifying some cortical areas as the supplementary motor area, premotor area, primary motor area in case of motor function; primary somatosensitive area in case of sensory; frontal aye field and primary visual or striate cortex in case of visual function; primary auditive cortex or transverse gyrus of Heschl in case of auditive function; and Broca's and Wernicke's area in case of language and comprehension.

As known by anatomy the supplementary motor, premotor area and frontal eye field are located respectively along the most medial, posterior and lateral portion of the superior 
frontal and immediately facing the precentral gyrus, seen in axial and sagittal T2-weighted slices. The central sulcus of Rolando and therefore the primary motor area (precentral gyrus), and primary somatosensitive area (postcentral gyrus) located just in front and behind it can be easily located by selecting the most rostral axial T2-weighted slices.

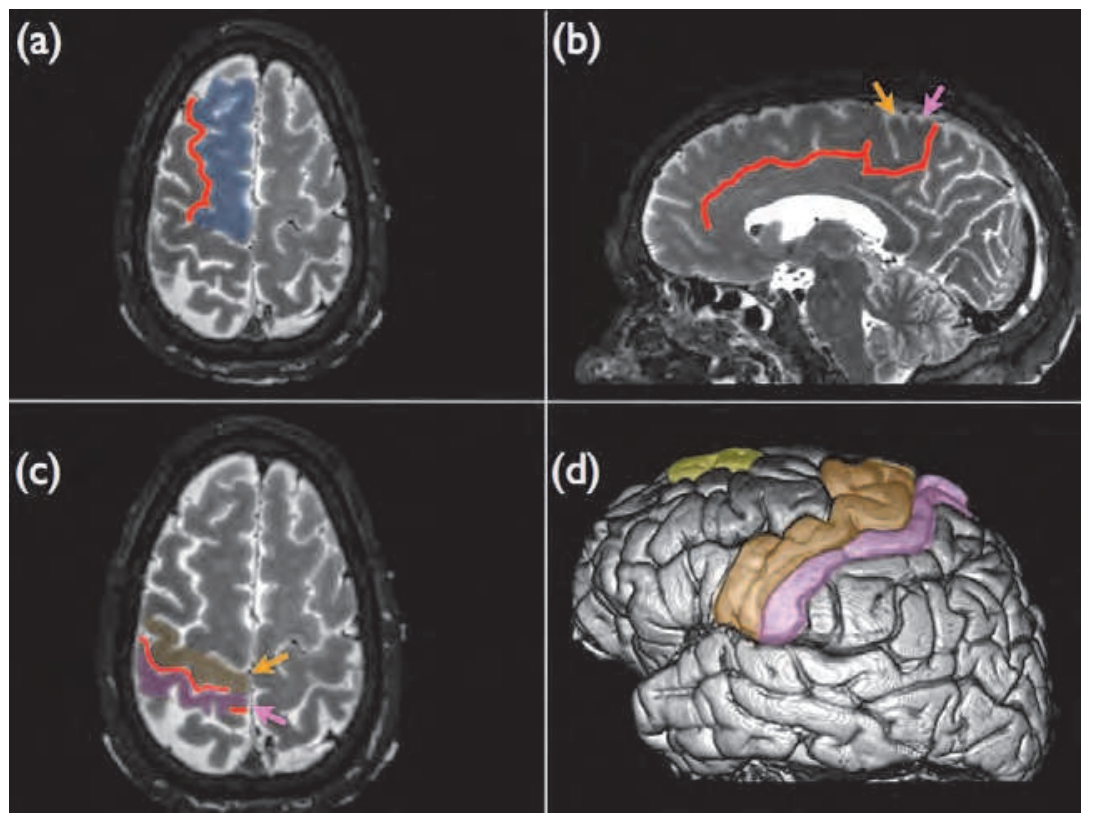

Fig. 3. (a) Axial T2 - weighted MRI slice where the superior frontal gyrus and superior frontal sulcus are marked in blue and red respectively; (b) midline sagittal T2 - weighted slice with the cingulate sulcus marked in red, showing the primary motor and somatosensitive areas through orange and pink arrows; (c) Axial T2 - weighted MRI slice where the primary motor and somatosensitive areas are marked in orange and pink, and the central sulcus in red; (d) 3D reconstruction from a T2 - weighted multislice study, with the supplementary motor, primary motor and somatosensitive areas in yellow, orange and pink respectively

The central sulcus is a good reference for the location of the motor homunculus, since despite the mass effect is always present and allows the preoperative study of the motor areas. In a parafalcian sagittal section, the Rolandic cortex (motor and somatosensory areas) is easily identifiable by following the superior cingulate gyrus and then to its end point (ascendent or marginal gyrus). The rolandic cortex is just anterior to this groove. In more lateral sagittal images, drawing a perpendicular line upward from the posterior corner of the insular triangle can easily identify the precentral sulcus (Fig.3).

Broca's area is located in the dominant hemisphere in the opercular and triangular parts of the inferior frontal gyrus, which can be easily visualized in a lateral sagittal slice (Gallucci, 2007; Ribas, 2010). This area is visible on coronal sections of the brain, when this section, perpendicular to the commissural plane, is located within 15 or $20 \mathrm{~mm}$ in front of the anterior commissure. 
In the same sagittal slice, Wernicke Area is located over the reverse C - shaped convolution constituted by the connection of the supramarginal and superior temporal gyri that encircles the posterior end of the sylvian fissure (Fig. 4).

The primary auditive cortex is located in the transverse gyrus of Heschl, which can be located using the sylvian fissure and superior temporal sulcus as the main landmarks. In a coronal slice at a point where the body of the lateral ventricles becomes into the atrium, the transverse gyrus shows a typical convex image between the superior temporal and lateral sulcus (Fig.5).

The main landmarks to locate the primary visual area are the calcarine and parieto occipital fissures, and remain well outlined on a midline sagittal section of the brain.

Despite all these landmarks and reference points to locate the brain eloquent areas, in the clinical practice this fact can be more difficult due to the peritumoral edema and mass effect, which usually distorts the normal brain anatomy. In this sense other imaging techniques have appeared to solve this problem.

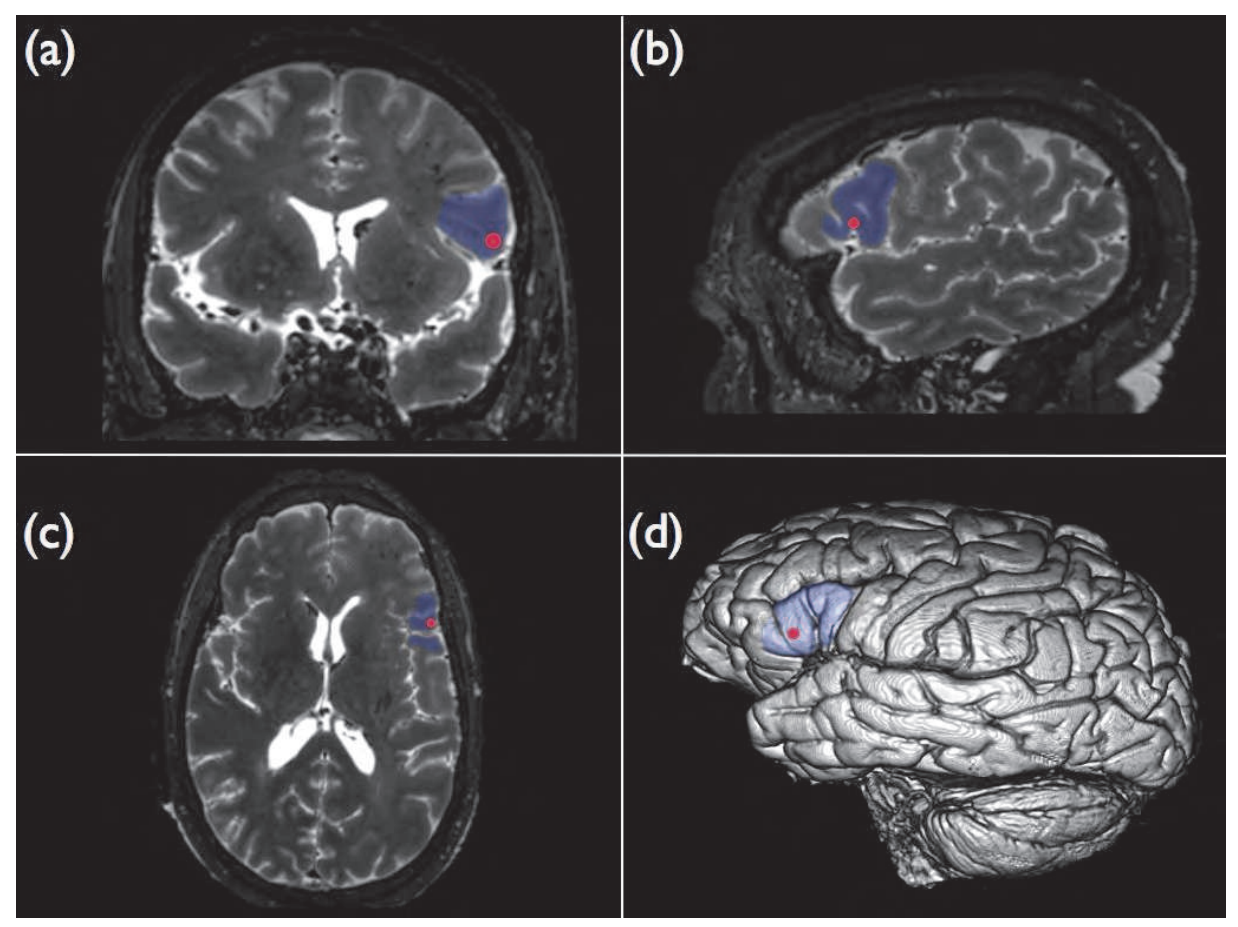

Fig. 4. (a) Coronal T2 - weighted MRI slice $15 \mathrm{~mm}$ rostral from the anterior commissure with the pars opercularis in blue and a red dot as reference for the next slices; (b) sagittal T2 weighted MRI slice from the red dot of the coronal slice and part of pars triangularis and pars opercularis in blue; (c) axial T2 - weighted MRI slice with Broca's area in blue; (d) 3D reconstruction from a T2 - weighted multislice study, where Broca's area can be identified in blue 


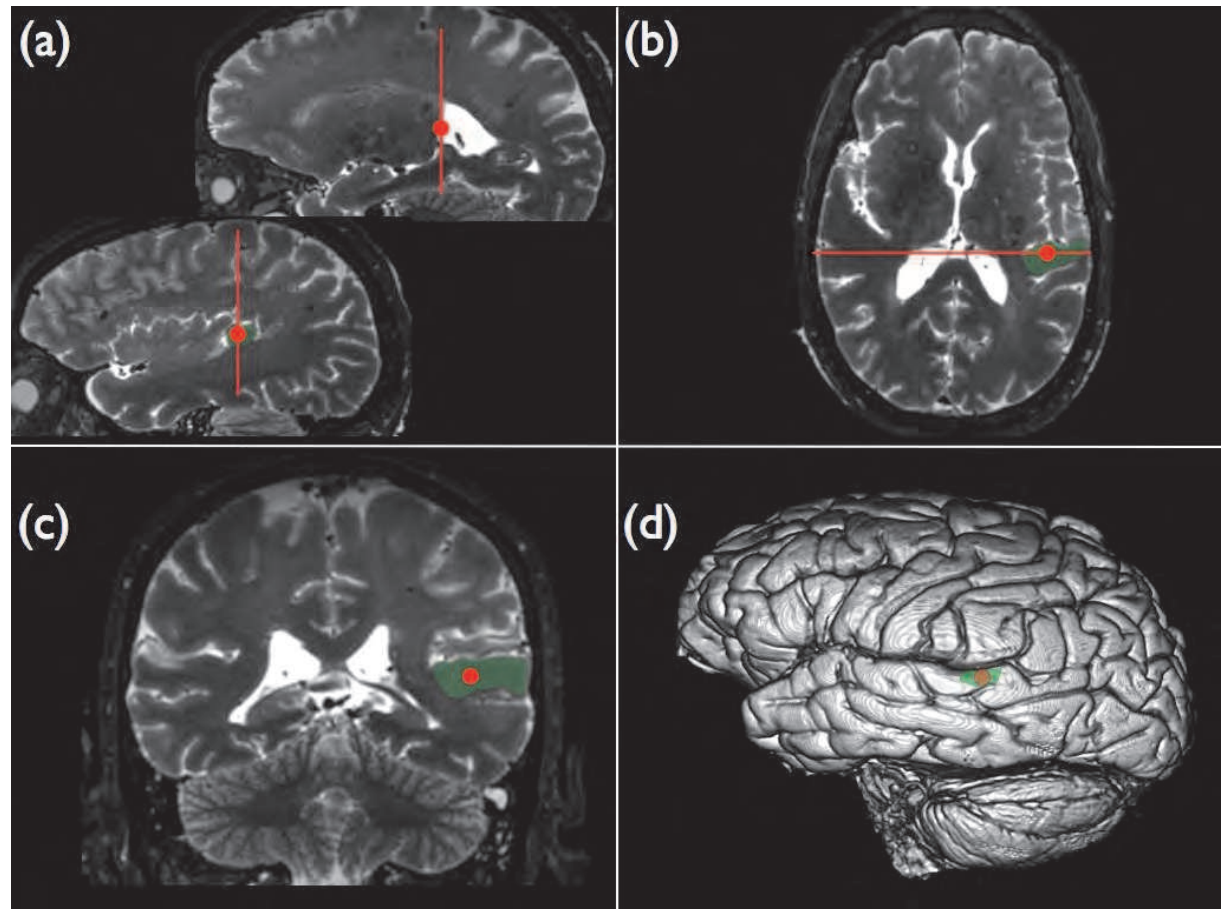

Fig. 5. (a) Sagittal T2 - weighted MRI slice at a point where the body of the lateral ventricles becomes into the atrium and its lateral translation till reaching the transverse gyrus of Heschl in green; (b) axial T2 - weighted MRI slice with transverse gyrus of Heschl in green; (c) coronal T2 - weighted MRI slice at a point where the body of the lateral ventricles becomes into the atrium and the transverse gyrus of Heschl can be easily located in green; (d) 3D reconstruction from a T2 - weighted multislice study, where primary auditive cortex can be identified in green

\subsubsection{Metabolic imaging}

These modern imaging modalities have been developed with the aim of offering information about metabolic changes in the core of the tumor and also in the supposed infiltrated surrounding gray and white matter. This information is extremely helpful in cases of tumors in eloquent areas due to the planning for extent of resection.

Classical anatomic MRI sequences are highly sensitive but nonspecific, cannot differentiate tumor and treatment effect, are unable to assess early therapy failure and predict clinical outcome. In this sense, neuroimaging should improve in characterizing brain tumor biology and assessing therapeutic response. Advances in neuroimaging have allowed a transition from anatomy to biology through physiology. Two new imaging modalities are being reviewed: perfusion - weighted imaging and proton MR spectroscopy.

\subsection{Perfusion - weighted Imaging}

This imaging modality offers a relative measure of blood volume and an indirect degree of angiogenesis inside a tumor (Fig.6). Perfusion MR provides additional information to 
standard gadolinium enhanced sequences. Gadolinium enhancement in standard contrast sequences shows blood-brain barrier breakdown, while in case of perfusion MR, it offers information about tumor tissue perfusion through its microvasculature (Kim \& Zee, 2007). When a patient with a brain tumor receives an injection of gadolinium, a T2 - weighted image is used to measure the decrease in signal intensity of the perfused tissue volume, and different perfusion parameters as time to peak, mean transit time, relative cerebral blood volume (rCBV), and regional cerebral blood flow (rCBF) can be calculated (Kim \& Zee, 2007; Ostergaard, 2005). The rCBV reveals significantly lower values in low - grade gliomas than in high grade gliomas, in fact, several studies have found a significant correlation between the rCBV inside a tumor and glioma grading. However, it can not differentiate yet between grade I and II, grade II and III or grade III and IV neoplasms.

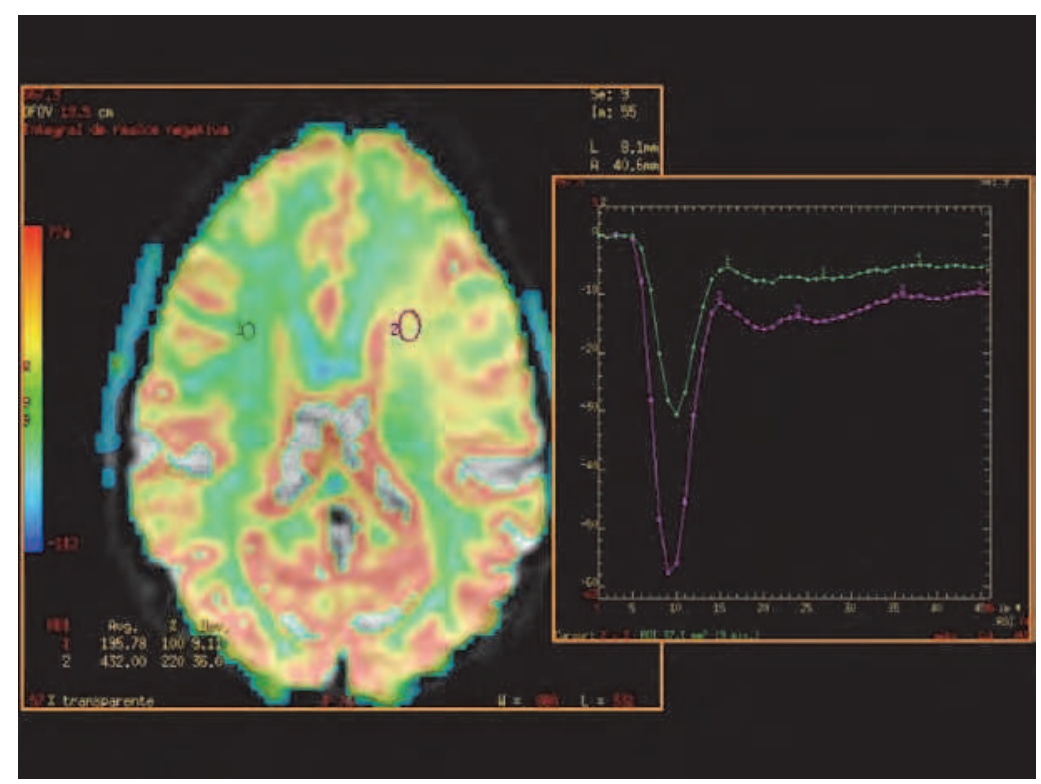

Fig. 6. MRI sequence showing the color map from the perfusion analysis and the increase of perfusion in a point of left centrum semiovale

Despite the general information offered by this imaging modality, one of the main interest in surgical planning, is the fact that $\mathrm{rCBV}$ values can be found significative higher in areas surrounding the contrast enhanced mass, where other imaging modalities do not show alterations due to the artifact of the edema.

\subsection{Proton MR Spectroscopy}

Spectroscopy allows the detection of heterogeneous metabolic areas within a tumor, which may be present beyond the margins that show classic MRI sequences. This technique detects increases of choline (Cho) in glial lesions, which represents cell proliferation, decreased concentrations of $\mathrm{N}$ - acetylaspartate (NAA) denoting loss of neurons as tumor cells replace them, and especially in high-grade gliomas increase of lactate (marker of necrosis) and increase of mobile lipids (Fig.7). 


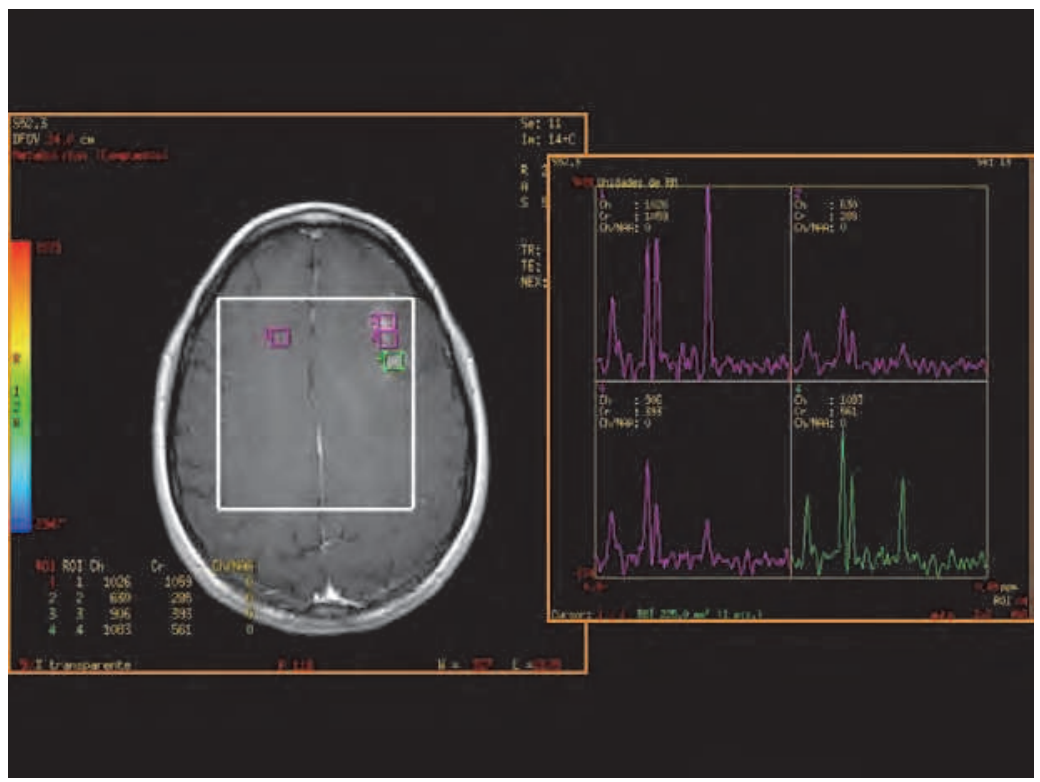

Fig. 7. Proton MR spectroscopy analysis of four different voxels. The numbers 2,3 and 4 are compared with number 1 , a control in the healthy side

\subsection{Preoperative study}

The classic, anatomic and metabolic MR modalities are really helpful in diagnosing, localizing and characterizing a brain tumor. However, for surgical planning of a brain tumor in a highly functional area, the most important point is to perform a 3D reconstruction of the brain where the surgeon can easily recognize the tumor volume, the involved gyri and the subcortical white matter tracts displaced and/or affected. For this purpose some imaging modalities have been developed: Functional MRI, DTI - based tractography and 3D anatomic sequences.

\subsubsection{Functional imaging}

Its main goal is the preoperative localization of eloquent areas prior to tumor resection to minimize intraoperative damage to these brain areas and thereby to reduce postoperative morbidity.

Functional MRI (fMRI) looks at changes in cerebral blood flow, and more specifically, in the balance of oxy and deoxyhemoglobin in the capillary circulation, and it can be viewed dynamically as a result of stimulation and performing tasks that are marked. Neuronal activation is measured indirectly through changes in blood oxygenation - dependent signal (BOLD). There is wide evidence that the location of motor and language areas by this technique is really helpful for preoperative planning in addition to being a noninvasive technique. A concept we should not forget is that it remains a technique that without the ability to distinguish between the various components of any specific functional response, and therefore may overlap different functional areas of brain processes, magnifying certain eloquent regions. 
In surgical routine the cortical regions mainly mapped through fMRI are speech, comprehension, motor, sensitive, visual and memory areas (Fig.8). In order to detect changes in blood oxygenation in speech areas, the patient is asked to pronounce different words or phrases. The chosen task in case of comprehension mapping consists on asking the patient to listen to and think about different phrases. If the chosen task is for motor mapping, patients are asked to move different parts of their bodies repeating the movements at least during 50 or 60 seconds in order to try to become these movements in a routine with the aim of avoiding blood oxygenation changes in prefrontal areas, cerebellum and basal ganglia. When the desired mapping is for sensory areas, the task will consist on pressing different parts of the body of the patient through a mechanic system. Memory mapping is performed through the task called "walking in my city", asking the patient to mentally remember all the details in a walk between two chosen points in his city.

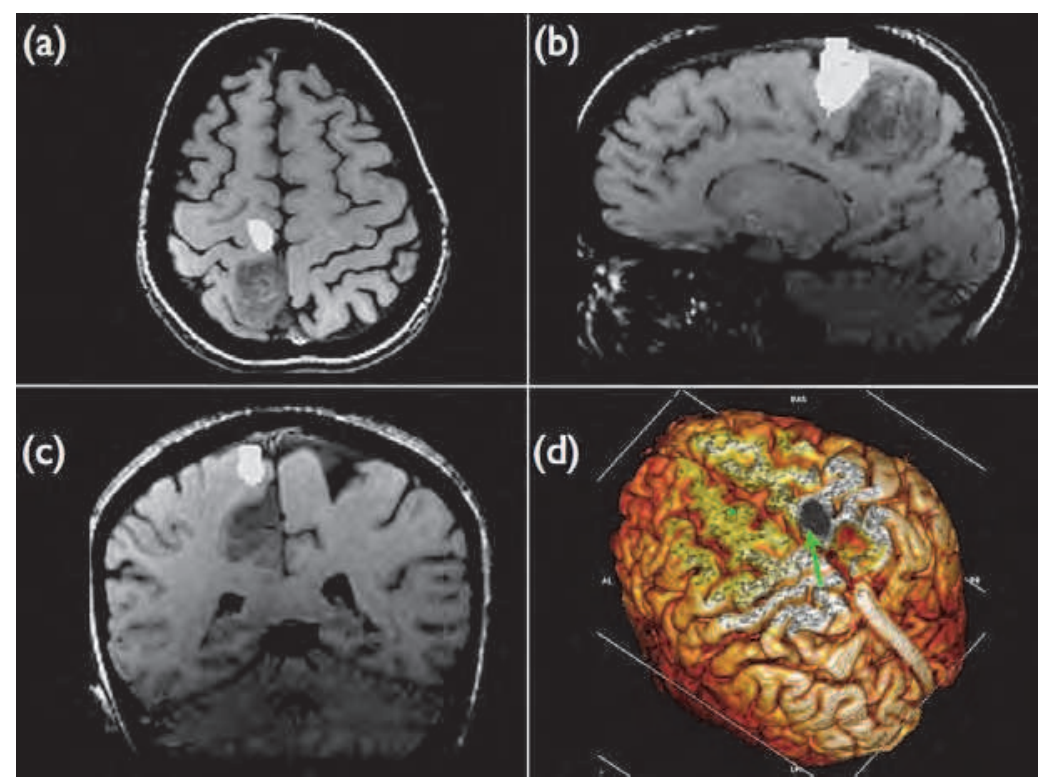

Fig. 8. Axial, sagittal and coronal T2 - weighted MRI slices (a), (b), (c), where a intrinsic tumor is located in the postcentral area and the activation from the fMRI for the leg (white stain) is located just anterior to the tumor; (d) 3D reconstruction from a T1 - weighted multislice study, where the three - dimensional relation between the activated area and the tumor are easily recognized

\subsubsection{DTI - based tractography}

Diffusion tensor imaging (DTI) is able to detect the characteristics of water diffusion in tissue distribution and display particular dimensional Brownian motion. The diffusion of water particles is facilitated by the ordered distribution of neural fibers through the membranes covering the axons. This imaging modality allows displaying the white matter fiber tracts by selecting a place where they can be easily visualized and then performing a three - dimensional reconstruction of them. DTI allows the visualization of bundles of axons in the human brain, by selecting regions with the same values of Fractional Anisotropy as 
"seed" for the start of production of tracts in the three - dimensional space (Berger \& Hadjipanayis, 2007).

Craniocaudal direction fascicles contain not only motor tracts, but also consist on somatosensory tracts that will be visible depending on where the seed is placed. The motor and somatosensory fascicles are located differently in the brainstem, at cerebral peduncle the first, and medial lemniscus the second. Following this reasoning, tractography can become a great tool for locating the primary motor area using the same regions with fractional anisotropy values when deciding where to place the seed.

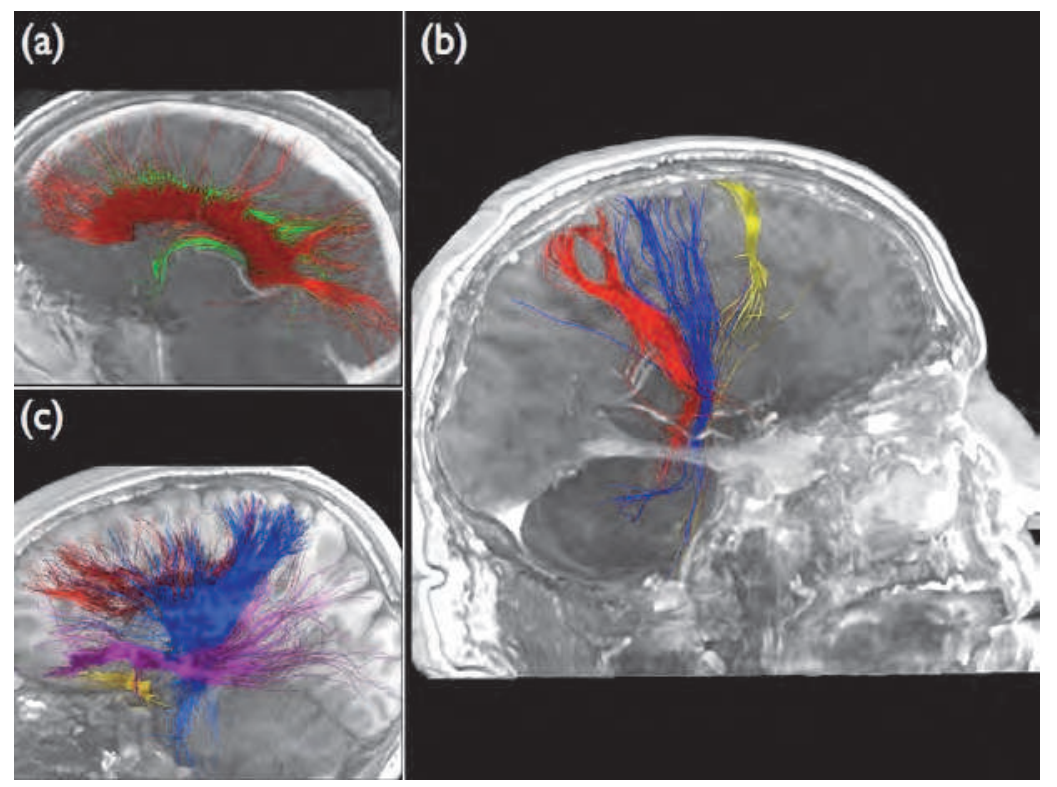

Fig. 9. (a) DTI - based tractography of the corpus callosum, cingulum and fornix in red and green; (b) DTI - based tractography showing the somatosensitive, motor and frontopontine fiber bundles of the internal capsule in red, blue and yellow respectively; (c) DTI - based tractography of uncinate, occipitofrontal, internal capsule and corpus callosum in yellow, purple, blue and red respectively

Tractography provides information about the normal course, the displacement or disruption of white matter tracts around the tumor, as well as damage to these tracts due to vasogenic edema or tumor infiltration. In Neurosurgery tractography is starting being used not only to understand the subcortical relation between the tumor and clinically relevant white matter fiber tracts, but also with the intention of localizing the motor and association pathways starting from their corresponding cortical areas, which are the neurons that leave these axons (Fig.9). In this sense DTI - based tractography can result of great help in cases in which fMRI cannot be easily performed. One of the biggest problems in functional MRI is the large number of variations of the venous circulation and the variety of hemodynamic responses that a brain in pathological conditions presents. Moreover, in patients with focal deficits or cognitive processing of tasks requiring fMRI is often very laborious and can not be done in many cases due to the inability of the patient (Basser, 2000). In these cases DTI is 
a really helpful tool to distinguish different cortical areas. It is important to have in mind that tractography is also limited in areas where tracts cross tumors or peritumoral vasogenic edema, so this fact should be noticed when deciding the place for the seed.

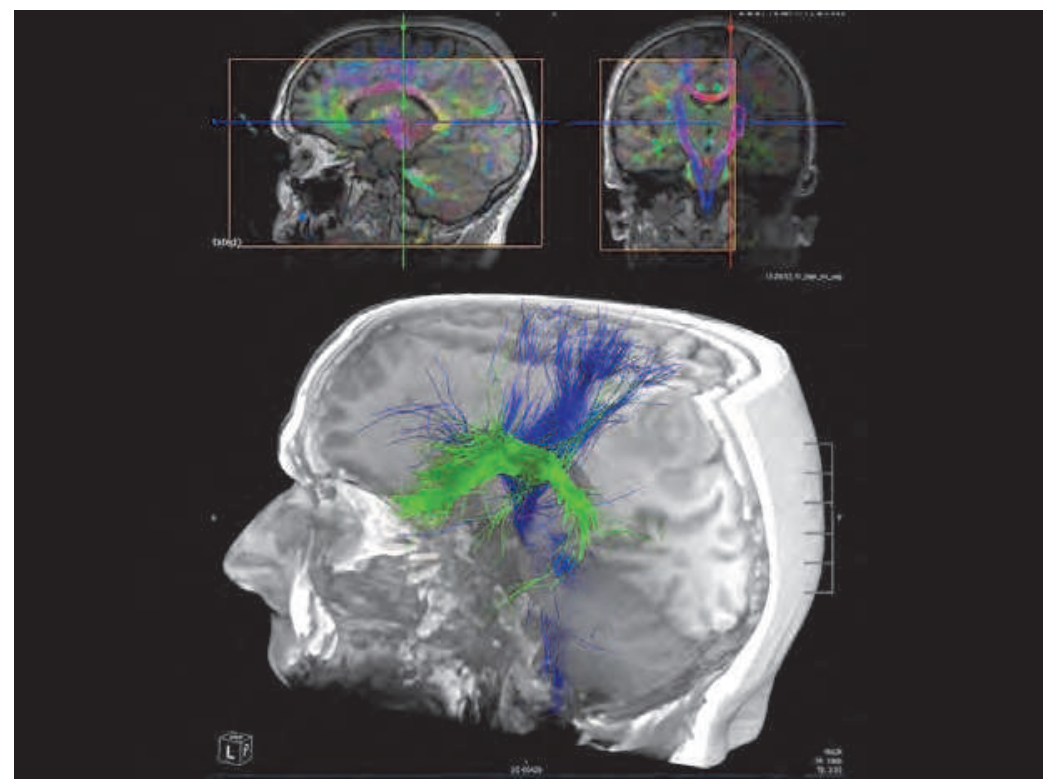

Fig. 10. Tensor preparation and seed placement of the internal capsule (pink boxes in sagittal and coronal slices in the color map), and 3D reconstruction showing the superior longitudinal fasciculus and internal capsule in green and blue

A great number of fascicles can be visualizes through DTI - based tractography, but in the neurosurgical practice the main studied fiber bundles are the corticospinal tract that contain motor and somatosensory fibers from the central lobe, passing through the corona radiate to join the internal capsule where the sensory part incorporates from the thalamic nuclei, and the motor fibers will lead to the cerebral peduncle; the superior longitudinal fascicle contains the arcuate fasciculus, which joins Broca's and Wernicke's area, resulting of great interest in cases of tumors located in the surroundings of the inferior frontal, angular and superior temporal gyri; and the optic radiations are also of great interest dealing with tumors not only in the occipital lobe, but also deep in temporal and parietal lobes (Fig.10).

\subsubsection{Three - dimensional anatomic sequences}

Once localized the tumor, marked the eloquent cortical areas and fiber tracked the subcortical white matter related to the tumor, next step consists on integrating all these data over a 3D anatomic sequence. The best sequence to perform this step is the one known as Three Dimensional Magnetization Prepared Rapid Acquisition Gradient Echo (3D MPRAGE). A 3D T2 - weighted image can be also helpful in some cases because it also offers a great accuracy of sulci and gyri (Fig.11).

These sequences offer the chance of performing a 3D reconstruction of the brain, being very helpful to plan the surgical approach and transulcal microsurgical technique. 

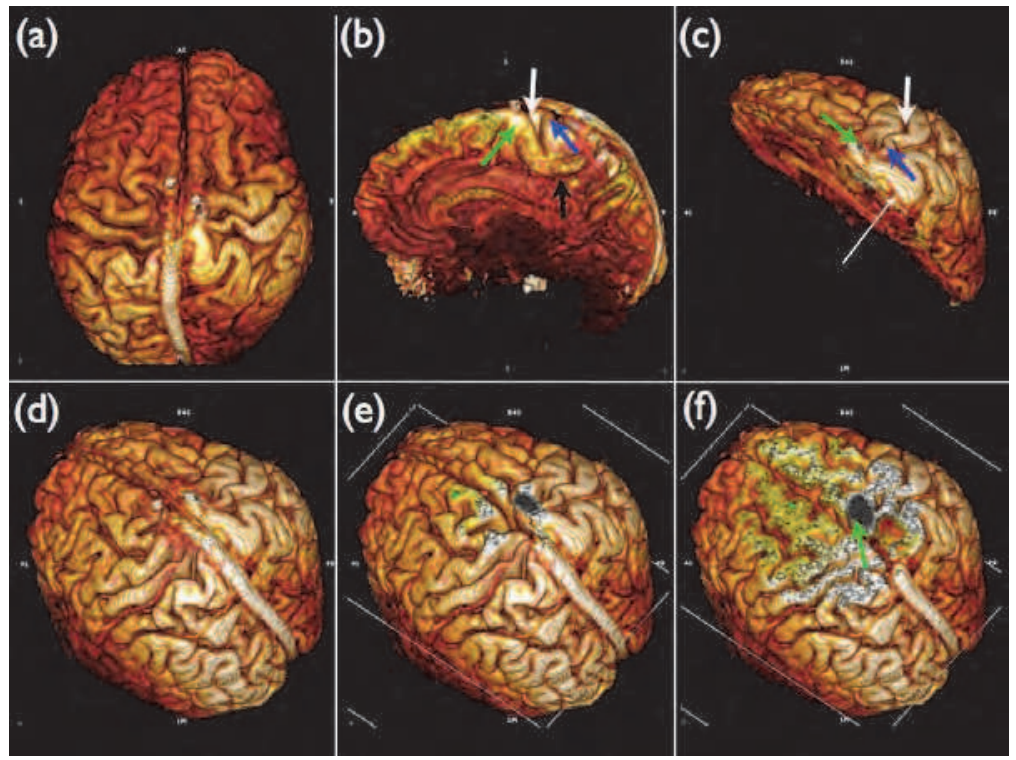

Fig. 11. Different views of a 3D reconstruction from a T1 - weighted multislice study showing the relation of a postcentral tumor (swollen gyrus marked with a thin white arrow in (c)), the precentral gyrus marked with a green arrow in (b) and (c), postcentral gyrus marked through a blue arrow in (c), and the fMRI activation for the left leg showed through a grey stain in (f)

\subsection{Intraoperative neuronavigation}

A neuronavigator is a passive intraoperative position-sensing system. Its main task is to encode the six-dimensional position of a surgical tool, to register the coordinate frame of the position sensor with some preoperative three-dimensional medical images using anatomic landmarks or fiducials, and thus to display in real time the location of the surgical tool on preoperative images. To establish a relation between a coordinate in physical space and its corresponding coordinate in image space a computer workstation calculates a transformation matrix (Vahala et al., 2001).

This technology allows the surgeon implementing all the chosen imaging modalities over a system of brain navigation, having stereotactic frameless control of the theoric extent of resection, and the relative location of different brain structures, of course including the tumor.

Image-assisted surgery is continuously being developed to help neurosurgeons to practice surgery on brain tumors more safely and effectively. Neuronavigation allows surgeons to locate intra-axial brain tumors more accurately choosing the best path to the lesion. All intraoperative navigation systems are fed with imaging studies, either a CT or MRI. The choice of appropriate images is really important, as for example, low-grade gliomas are best defined by T2-weighted sequences, whereas high-grade primary tumors are best seen on a T1 contrast (Berger \& Hadjipanayis, 2007).

The competency to combine functional studies of the diffusion tensor is a tool that provides relevant data when intraoperative planning surgical approach. 
Integration of preoperative functional data extracted from fMRI, known as functional neuronavigation, has become a routine practice in surgery of lesions adjacent to eloquent areas of the human brain, in order to avoid postoperative deficits (Fig.12). But in addition to eloquent cortical areas, deep structures, as are the bundles of white matter fibers connecting these areas should also be respected during surgery. The images produced by the tractography help the surgeon to understand the connections and the location of the tracts during surgery, and their relationship with cortical anatomy and brain disorders.

These new additions to the surgical planning are a major consumer of time spent on analysis and data processing. The accuracy of the information provided by tractography depends largely on the experience of the individual processing. Since the introduction of these technologies in the routine surgical procedure for brain tumors, the extent of resection avoiding surgical morbidity has improved exponentially.

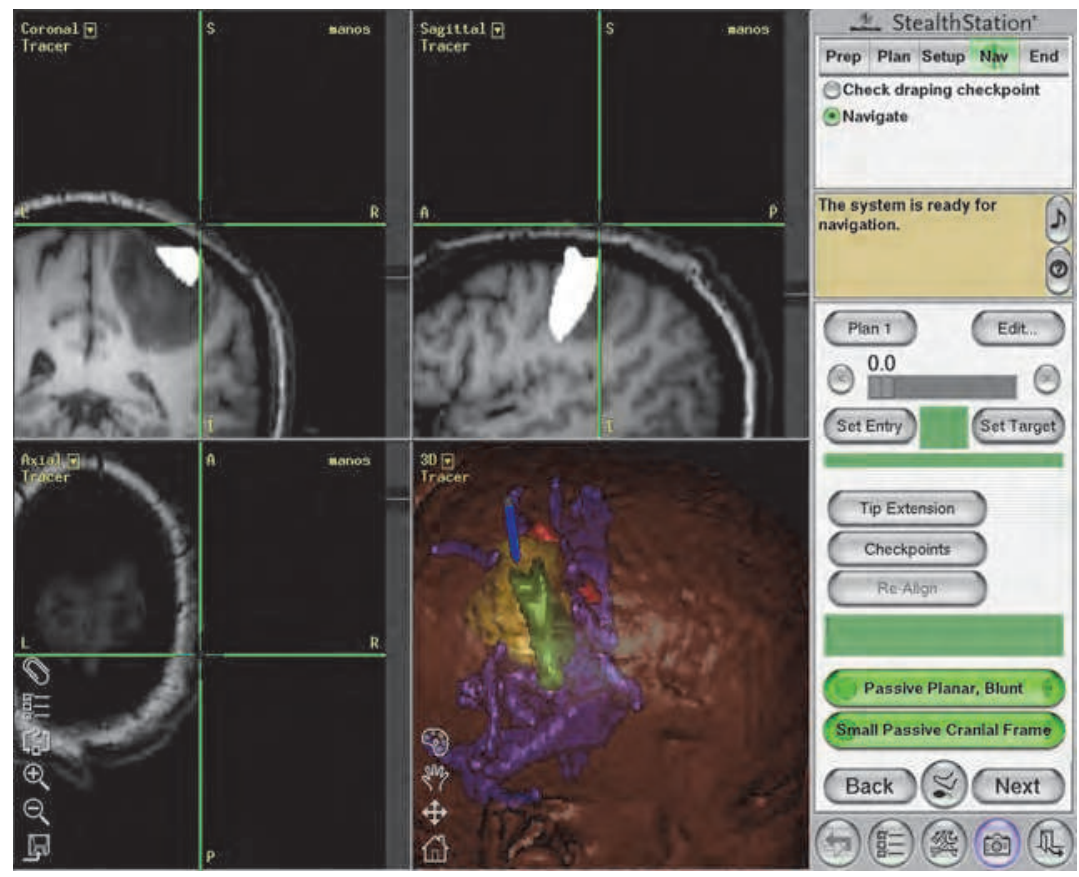

Fig. 12. Screenshot from the intraoperative neuronavigator showing the three orthogonal slices showing the white stain from the arm activation of fMRI and its relation with the tumor in precentral gyrus, and the 3D reconstruction where it is easy to visualize the tumor, arm and leg activations and internal capsule motor projections, in yellow, green, red and blue respectively

\subsection{Intraoperative imaging modalities}

The role of radical surgery in the management of glial - type brain tumors is still controversial. In fact, there is no scientific evidence that a greater extent of resection is associated with a better prognosis. Class II data show that the extent of resection for malignant gliomas improves survival and in case of low - grade gliomas, improves survival 
and time to tumor recurrence (Keles et al., 2001). This is the main reason why until new treatments for glial tumors are developed, the cytoreductive treatment provided by surgery will maintain its value. The price to pay for radical resection may be an increase in morbidity. In this sense, different intraoperative imaging modalities have been incorporated to the clinical practice with the ultimate objective being to optimize the resection limits; to extend them to the maximum while minimizing the eventual associated morbidity (Gonzalez-Darder et al., 2010).

The main limitation of neuronavigation is its reliance on preoperative images. Upon opening the skull and dura mater, movements inevitably occur, making data derived from these images unreliable and then loosing accuracy. Only intraoperative imaging is able to offer the updated information needed to maintain accurate navigation during the surgical procedure. These images are useful to confirm that tumor resection has been completed, a fact often not verifiable under the surgical microscope vision (Schulder \& Carmel, 2003).

\subsubsection{Intraoperative magnetic resonance}

Intraoperative magnetic resonance imaging (iMRI) is a technology not largely employed yet due to its high cost and its special needs in the operating room (OR). An OR must be previously designed to host an iMRI. The complexity of the OR setup is greater for the use of iMRI, and safety as well as equipment details increase proportionally to the field magnetic strength.

How iMRI may influence quality of life and survival remains to be studied yet, but it seems that selected patients with low and high - grade gliomas will clearly benefit from the use of intraoperative imaging techniques as iMRI, due to the ability of them to offer in real time a direct control of the extent of resection, the relative location of the cortical and subcortical eloquent regions with the tumor (Nimsky et al., 2005).

\subsubsection{Intraoperative ultrasound}

Intraoperative MRI technical pitfalls have let to the development of intraoperative ultrasonography in many centers. Ideally all brain tumors are at least partially hyper echogenic. Diffuse calcifications inside lesions produces stronger echo patterns, while cysts or areas of necrosis are hypo echogenic. The local invasion of gliomas tends to appear as intermediate echogenicity. The edema can also be distinguished from the surrounding healthy parenchyma.

The main limitation of ultrasound is image resolution and the correlation of preoperative MRI scans and intraoperative ultrasonography images. This problem has been partially solved with the aim of modern neuronavigation systems, which allow overlapping of real time ultrasound images with preoperative MRI data (Berger \& Hadjipanayis, 2007).

\subsubsection{Fluorescence guided resection with $\mathbf{5}$ - aminolevulinic acid}

This technique allows the visualization of malignant tissue during surgery for malignant glioma (grades III and IV WHO). Tumor resection guided by fluorescence involves giving the patient a natural precursor, 5-aminolevulinic acid (5-ALA $\mathrm{HCl}$ ), which is taken up by cells of malignant gliomas and, when summed, becomes a fluorescent substance. Thus, by applying a special light during surgery, the malignant cells are stained red offering the surgeon a clear distinction between the healthy and which are not, letting increasing the extent of tumor resection, minimizing brain damage (Stummer at al., 2006)(Fig.13). 


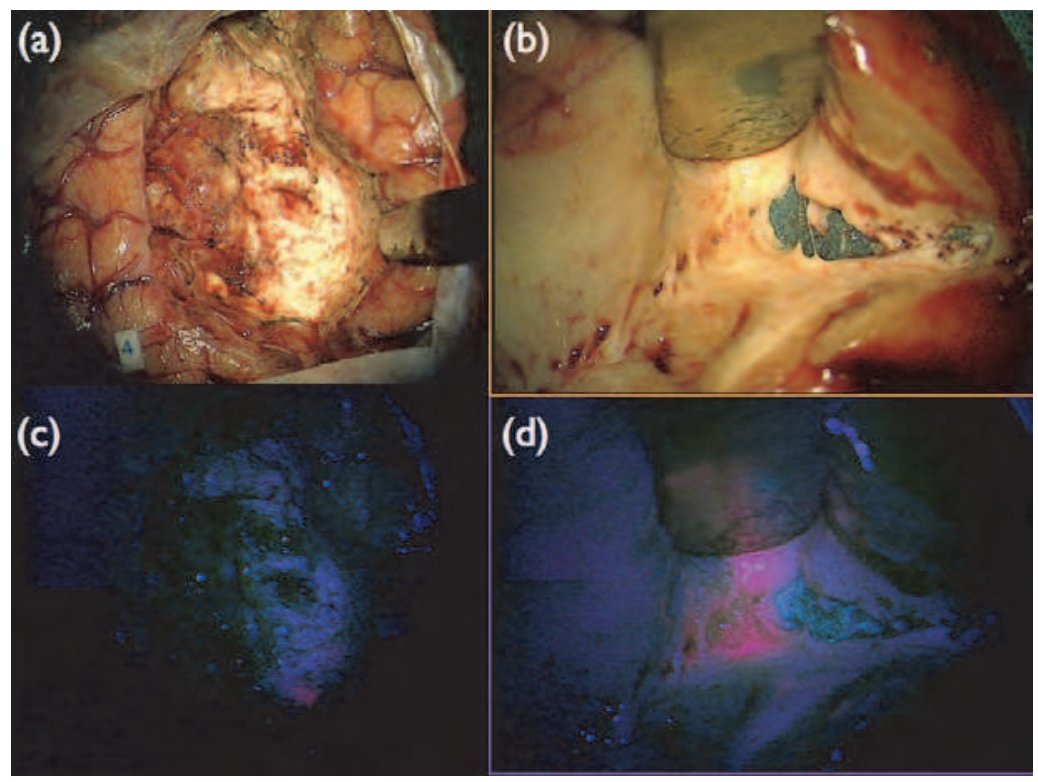

Fig. 13. Intraoperative views from brain tumor resection cavity and tumoral piece resected under the white light of the microscope (a) and (b); views of the same pieces under fluorescence special light where it is possible to appreciate pink areas which show the invasion by tumoral cells

\subsection{Postoperative follow - up}

Obviously the best imaging technique to evaluate the surgical resection of a brain tumor is MRI. The time between imaging controls to update the evolution of a brain tumor after surgery will depend on the initial extent of resection and the tumor grade. The sequences employed will be the same used preoperatively, as T1 - weighted with contrast and T2 weighted imaging, flair, perfusion - weighted imaging and spectroscopy.

An important point in gliomas follow - up is to perform an early postoperative image control, in order to use it as the starting point to evaluate possible future complications and tumor recurrences, and also to evaluate tumoral remains. In this sense, although the contrast enhanced T1 - weighted imaging, the diffusion - weighted has emerged as a really helpful sequence. This early image control should be performed in the first 48 hours after the surgical procedure.

Acute cellular damage in the surrounding parenchyma after brain tumor resection occurs due to direct surgical trauma, retraction and vascular injury, and devascularization of tumor. This cellular damage results in acute intracellular swelling and decrease in the surrounding extracellular space can lead to reduced diffusion in the brain. This fact remains really important in the evaluation of an early postoperative MRI. These areas of immediate postoperative diffusion abnormality invariably undergo a phase of contrast enhancement on routine anatomic images that can be easily misinterpreted as tumor recurrence. This is the reason why an early detection (24-48 $\mathrm{h}$ post - surgery) of these areas let the surgeon differentiate a tumor rest from a resection ischemic damage. 


\section{Functional mapping}

The goal of neurophysiological techniques in the identification of eloquent areas before and during brain tumor surgery is to achieve a better definition of eloquent areas in order to increase tumor resection with greater safety in avoiding damaging structures and potential functional irreversible neurological sequelae.

Locating eloquent areas is very useful considering the large interindividual anatomical variability, which is more pronounced in case of patients with space - occupying lesions that cause a distortion and displacement of anatomical structures, infiltrate functional areas and suffer neuronal plasticity phenomena.

There are several functional mapping techniques, which can be subdivided in two major groups depending when they are performed: preoperative and intraoperative techniques.

\subsection{Preoperative mapping}

\subsubsection{Navigated transcranial magnetic stimulation}

Transcranial Magnetic Stimulation (TMS) is based on the stimulation or inhibition of neuronal activity through a magnetic field centered over the skin. This technique was born as the evolution of Transcranial Electric Stimulation, improving the procedure due to the accuracy when applying the electrical flow.

The physical basis consists on a current discharged into an electromagnetic coil resting on the scalp, creating a magnetic field that induces a perpendicular electric field. The field produces an electric stimulus in the cortex. TMS may be used to map the motor, sensitive or language cortex. One of the best advantages of TMS is that it is the only noninvasive inhibition technique. Disadvantages include the risk of repetitive TMS causing seizures.

The main advantage of TMS over other non-invasive brain functional imaging techniques is that when a physiological response is evoked by stimulation of a cortical area, that specific area can be assumed to be directly responsible of evoking that response. With other imaging methods, it is only possible to detect and map a brain area that takes part in a previously decided task (Ruohonen \& Karhu, 2010). This fact assumes that TMS is much more selective in order to map the tumor surroundings, so as to avoid damaging them during surgery.

Although there has been relatively little reported use of TMS in surgical planning, recent technical advantages have introduced this technique in some neuronavigation systems, allowing neurosurgeons preoperatively mapping the desired cortex of their patients, and then introducing the data over a 3D brain anatomic reconstruction with the aim of improving the surgical planning (Tharin \& Golby, 2007). Neuronavigation of TMS enables the localization of eloquent cortex in patients with distorted anatomy as it is often found in brain tumors. Some advantages are present comparing with fMRI, as they are the fact that some tasks are difficult to be performed by patients with a brain tumor near the motor cortex, it avoids the artifacts given by fMRI and it can be monitored by neurosurgeons, allowing them to choose the cortex to be mapped (Forster, 2011).

\subsubsection{Somatosensory evoked potentials}

The SEP consist on the distal stimulation of afferent pathways with the aim of identifying short latency responses evoked from specific cortical areas (Fig.14). When stimulating the sciatic nerve distally, the response is detected on its specific area of the contralateral postcentral gyrus devoted to the representation of the lower extremity, while stimulation of the upper extremity will elicit a similar response from a different portion of the contralateral 
postcentral gyrus. With this approach, it is possible to elaborate a functional map of the somatosensory projection cortex in the postcentral gyrus.

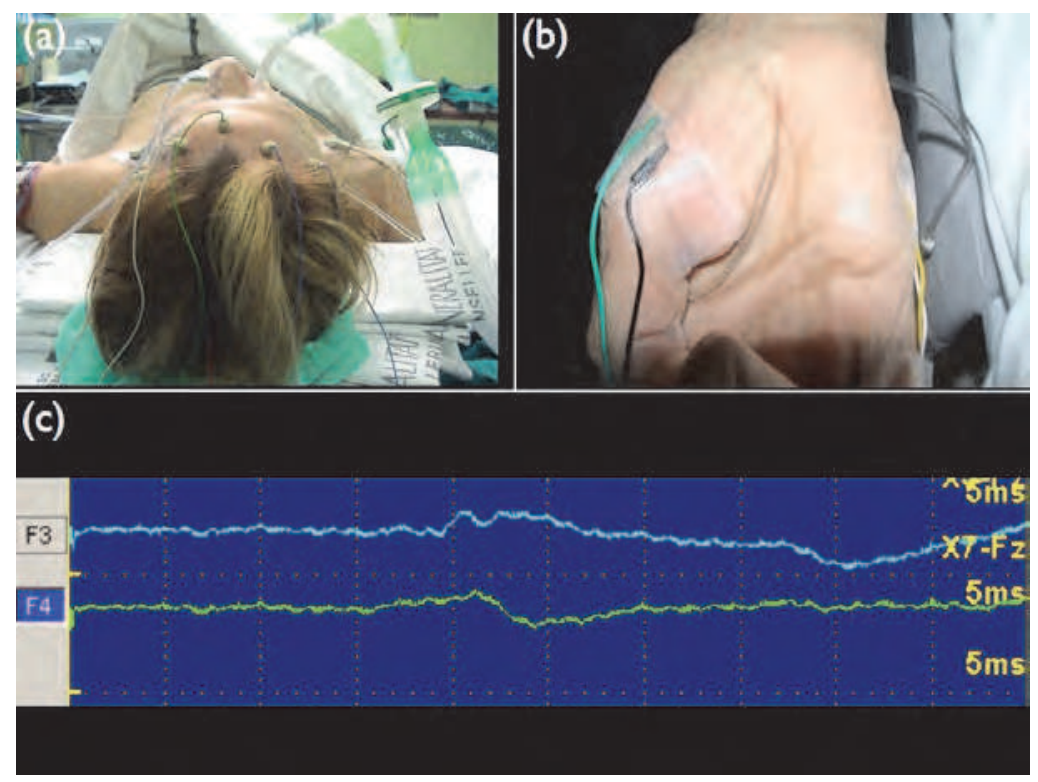

Fig. 14. (a) Scalp distribution of electrodes to receive the signal from the (b) median nerve stimulation in order to detect the (c) somatosensory evoked potentials register

The evoked potential method has been subsequently developed as a practical noninvasive clinical method to study conduction in the visual, auditory, and somatosensory systems. In the clinical practice this technique is used to check the somatosensory pathway integrity before, during and after surgery. Its preoperative status is of great importance in order to have a control to fastly detect any changes during surgery.

\subsection{Intraoperative mapping}

\subsubsection{General considerations for intraoperative brain mapping}

Different intraoperative mapping techniques have improved the results when resecting brain tumors near eloquent areas. Some of these techniques require the patient's collaboration in order to detect for example a speech arrest during Broca's Area stimulation.

Asleep craniotomy for brain tumors surgery is a useful technique in selected cases. When the tumor affects the area of language, the surgical procedure is performed under conscious sedation, in which the patient actively participates (object naming, counting and reading numbers) in much of the procedure. Therefore they must understand exactly the technique, the objectives, limitations and complications of the method. In these cases the aim consists on achieving conscious sedation, which means that the patient is asleep but responds to verbal commands. This sedation is objectified through an anesthetic depth monitor (Bispectral Index whose values should be between 70-80). With this level of sedation achieved good cooperation of the patient. 
If the patient's cooperation is not needed, there will be a total intravenous anesthesia preferably with appropriate depth level (BIS 40-50). Using propofol with remifentanil both in continuous intravenous infusion. The use of drugs that reduce or alter the potential such as neuromuscular relaxants, volatile anesthetics and benzodiazepines should be avoided.

However neuromuscular relaxants can be used to obtain a partial blockage, in such a way as to reduce an important part of the movement and to facilitate the surgical procedure, being able to obtain adequate monitoring (with an appropriate lock), except during the mapping. One of the possible complications of this method is the appearance of post-discharges associated with stimulation, such as to trigger a seizure. If a post-discharge appears over 30 $\mathrm{sec}$, it suggests the use of midazolam $2 \mathrm{mg}$ or lorazepam $2 \mathrm{mg}$, or low intravenous dose of barbiturates. It is also recommended the use of serum ringer at a lower temperature than ambient directly over the exposed cortex.

Other factors that influence the monitoring are: an adequate blood flow, intracranial pressure, optimal hematocrit, adequate ventilation and oxygenation and temperature of the patient.

\subsubsection{Somatosensory evoked potentials}

The somatosensory evoked potentials technique has exactly the same purpose intra than preoperatively, and has just been commented in the preoperative mapping section. Its main goal consists on monitoring the somatosensory pathways during surgery.

\subsubsection{Phase reversal of somatosensory evoked potentials technique: N20/P30 wave}

When dealing with cortico - subcortical tumors near the central sulcus, the fact of identifying this anatomical structure when opening the dura mater may result really complex due to the anatomical distortion caused by the tumor. For this purpose the N20 wave technique has been developed. SEP can identify primary areas of sensory and motor control (precentral and postcentral grooves).

The reverse phase of SEP is based on changes in the polarity of the dipole field generated by the cortical afferent pathway. The stimulus applied to a peripheral nerve generates an electric dipole on the postcentral gyrus. The polarity of this dipole changes on the adjacent precentral gyrus. Thus, a SEP (N20/P30) can register from the postcentral gyrus and a reverse image from the turn precentral ( $\left.\mathrm{P}^{\prime} 20 / \mathrm{N}^{\prime} 30\right)$.

Once performed the craniotomy and opened the dura, a strip of silicone that have set a variable number of electrodes ( 4 or 6 ) of platinum of 1-1.5 $\mathrm{mm}$ in diameter aligned in rows, with an interval of $0.5-1 \mathrm{~cm}$, will be placed over the cortex (Fig.15). The strip of electrodes should be placed across the alleged central sulcus, covering the area of the hand or foot at the sensorimotor gyri, with an angle of $15^{\circ}$ to the sagittal direction. For the SEP of the median nerve, the electrode should be placed in a cortical area between 3 and $8 \mathrm{~cm}$ from the midline. For the tibial nerve, the cortical area is limited to $0-3 \mathrm{~cm}$ from the midline.

In tumors affecting the central area close to the interhemispheric fissure, the posterior tibial nerve stimulation is used. This technique is more difficult to obtain, but very useful in these cases.

An electrical stimulator current of constant voltage will be employed for this purposes. Intensity should be gradually increased until a motor response in the first finger (median nerve) or the big toe (tibial nerve) appears. Once obtained the wave, it is easy to locate the central sulcus between the electrodes in which the phase reversal is given. 


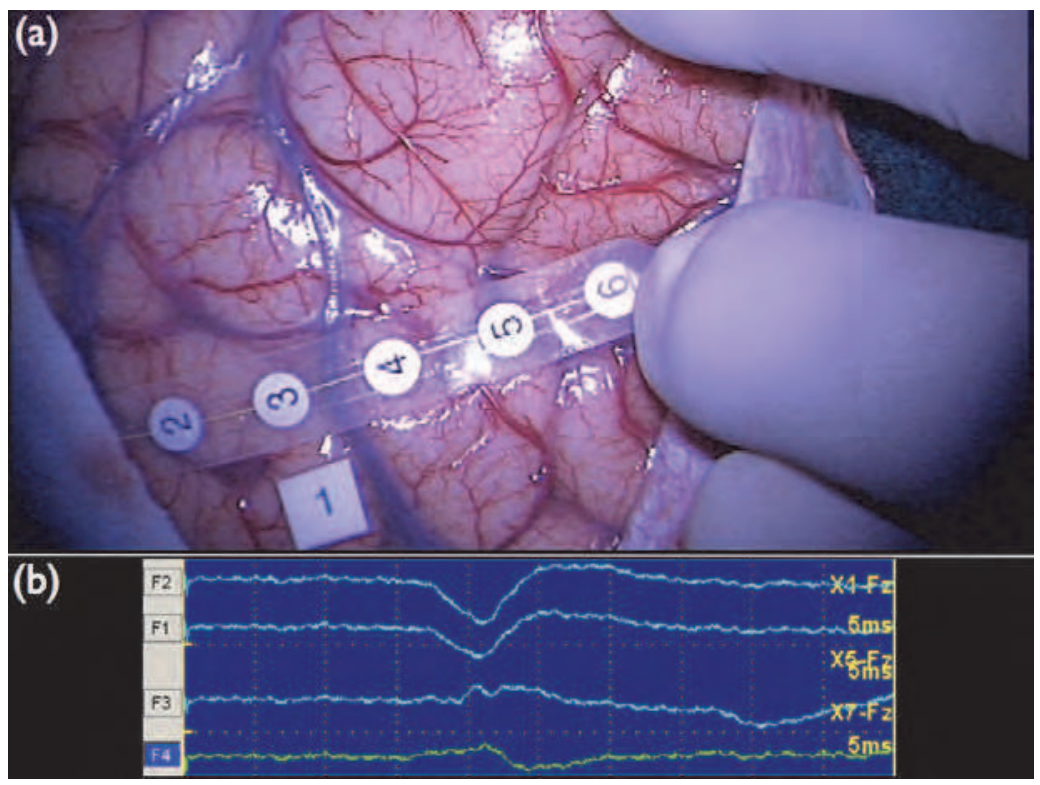

Fig. 15. (a) Flat strip over the brain surface to locate the central sulcus and the register (b) given after the stimulation where it is possible to appreciate the wave inversion between number 4 and 5 poles

\subsubsection{Direct cortical stimulation: looking for the functional gray mater}

This neurophysiological technique allows the surgeon localizing the functional areas with awake (language areas) or non - relaxed (motor areas) patients, through direct brain stimulation.

The technique of mapping by direct cortical electrical stimulation allows the realization of a functional map of the cerebral cortex exposed, both to identify areas that are functionally significant as areas that are not.

The neurosurgeon places the electrode at a small region of cortical area of the brain, and the stimulator from the computer applies a train of stimuli, which can result in neurological changes as patient movement or numbness or inhibit neurological function as speech arrest. When stimulation of a focal brain region produces any of the above symptoms not accompanied by a crisis or post-discharge, it is confirmed that the stimulated cortex region is important for brain function. The criteria for considering an area as eloquent, involves that a functional response occurs for at least three separate stimuli into a single cortical region (Pouratian, 2004).

The most employed technique for direct cortical stimulation is the repetitive bipolar cortical stimulation or Penfield technique (Fig.16). Bipolar stimulation technique of Penfield is based on the activation of the cortical circuit applying electrical pulses repeatedly.

For the stimulation is commonly used a bipolar electrode with carbon tips $5 \mathrm{~mm}$ apart, with a cable connected to an external stimulator that acts as a generator of continuous electrical power trains of biphasic pulse. To assess motor or sensory function, applying a train of 2-3 seconds long is enough, while assessing language function requires longer durations of 5-7 
seconds. Stimuli should not be encouraged two consecutive times in order to prevent post discharges and seizures. Three positive tests in the same location are enough to ensure that it is essential for language. Occasionally it is necessary to extend the duration of the train to 7-10 seconds to map the language, due to the complexity of questions and answers to the questions.

It is recommended to begin with a current of $1 \mathrm{~mA}$ from $2 \mathrm{~mA}$ to increase to the minimum intensity that produces a sensorimotor response (not higher than 8-10 mA). The procedure finishes by marking with sterile labels the cortex with positive response using the legend chosen by each surgeon, in order to avoid damaging these eloquent areas.

\subsubsection{Motor areas}

Registration can be done with the patient awake, determining the evoked movement by direct visualization or with the patient asleep using as control a continuous electromyographic recording.

Recording electrodes should be placed when the patient is anesthetized. The needle electrodes are subcutaneous monopolar and disposable $(12 \mathrm{~mm}$ or $20 \mathrm{~mm}$ in length, placed 2 separate needles 5-10 $\mathrm{mm}$ between them) and are placed in the muscle groups that correspond to the stimulated brain area (right / left, contralateral to the lesion; or bilateral, depending on the location of the tumor), for example, it is possible to monitor facial muscles (orbicularis oris) upper limb muscles (deltoid, biceps, extensor digitorum and short Abductor) and lower limb (femoral adductor, quadriceps, tibialis anterior and abductor). Best when it is possible to monitor the entire side of the body, with impedances below $5 \mathrm{~K} \Omega$.

The stimulus causes muscle jerking or tonic contractions that can start immediately to stimulation or after several seconds. In other regions the stimulation can cause inhibition of movement (when an awake patient is told to move his fingers, stimulation can slow or halt the movement), as supplementary motor cortex stimulation and other regions that can assume an integrative role in motor function.

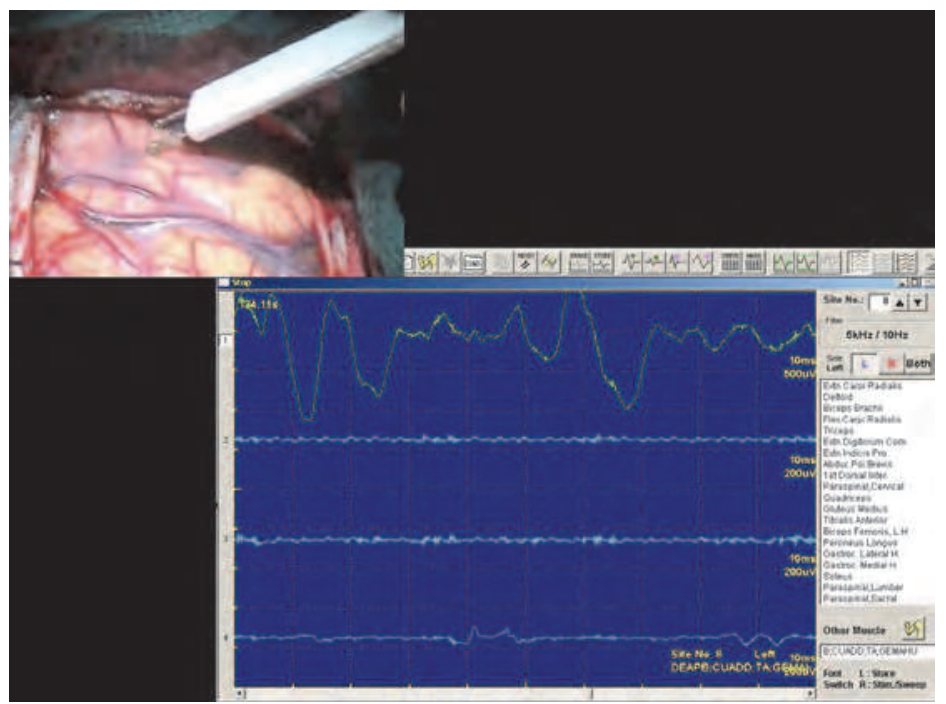

Fig. 16. Direct cortical stimulation and its register showing activation in the left cuadriceps 


\subsubsection{Sensitive areas}

It is performed with the patient awake and requires of his/her cooperation. The presence of sensory impairment is assessed by the patient, paresthesias in the contralateral regions of the body and occasionally on both sides of tongue or face, or both sides of the neck.

\subsubsection{Visual areas}

Visual areas are not usually intraoperatively mapped, but when performed, the patient must be awake and his cooperation is needed. It values the appearance in the contralateral visual field: lights, colors, shadows (when stimulated primary visual cortex). Stimulation in the visual association cortex may cause hallucinations.

In the event that a craniotomy is large enough, placing a blanket of subdural electrodes allows identifying and monitoring the bioelectric response of the visual region throughout the procedure, using visual stimuli from special glasses with LEDs. This technology facilitates the surgical procedure and avoids keeping the patient awake. This same methodology can be used to identify the primary auditory area.

\subsubsection{Language areas}

It is performed with the patient awake and requires their collaboration. The administration of drugs must be stopped at least 15 minutes before beginning the cortical stimulation.

It is desirable the involvement of a neuropsychologist for interpretation of different language errors caused by the stimulation. There should be a preoperative training about the tasks to be performed intraoperatively, and stop the monitoring in case the patient in a basal situation fails more than $25 \%$ of the tasks presented.

In a first step the sensorimotor response should be mapped to confirm a positive response. After confirming the response, next steps are:

a. Mapping cortical areas

b. Language sites whose answers after stimuli are known are mapped and produce the same inhibition (speech arrest, dysarthria or anomia).

A good beginning is naming and counting tasks (expressive language): asking the patient to count (1 to 10, again and again) or name objects presented visually (different test can be used, selected according to different variables such as frequency, familiarity, age of acquisition, and education). It helps to choose pictures of items that the patient can name quickly and easily in the test.

Phonetic Association: asking the patient to tell as many names beginning with the letters F, P and L (one minute for each letter).

Semantic association: the patient is asked to read the largest number of cars, fruits or animals.

Nomination of famous faces: showing the patient 50 pictures from famous people and 50 non - famous.

Object Naming.

Calculations simple: multiplication or subtraction.

c. Failures are coded and responsible areas are registered again with sterile labels:

Aphasia (loss of the ability to produce and / or comprehend language).

Phonemic paraphasias (changes in the articulation of one or more phonemes).

Semantic paraphasias (changes in the phonology of the word, weakness with the onset of the alterations).

Anomia (difficulty in retrieving words when speaking)

Perseverance (repeat previous items as the following items were submitted)

Speech arrest. 


\subsubsection{Electrocorticography}

The electrocorticography is a technique that involves recording cerebral bioelectrical activity through a strip of subdural electrodes. The procedure is more useful when is associated with direct cortical stimulation.

This neurophysiological procedure has the ability to detect both tumoral and irritative regions. It is often possible to identify directly the peri - rolandic region by the presence of a typical spindle shape of mu rolandic rhythm. It also allows detecting the stimulus artifact while stimulating the cortex, which verifies that cortex is being stimulated and electrocorticography is recorded.

The maximum stimulus intensity can be defined, and the incidence of post-discharges and seizures induced by electrical stimulation can be detected. The incidence of post-discharges or seizures following a train of stimuli during direct cortical stimulation, increases the possibility that the physiological effect of the train should be not localized due to the spread to a wider cortical region, fact that could cause a misinterpretation by the surgeon in case of not using the electrocorticography.

This technique is necessary when the intraoperative brain functional mapping is supposed to be longer than usual.

\subsubsection{Direct subcortical stimulation: extent of resection in the white matter}

Subcortical stimulation is a novel technique that has reached as importance as cortical mapping in brain tumor surgery. Its main indication remains when dealing with tumors with a subcortical extension in the surroundings or inside important white matter fiber bundles. The technical details and the tools employed are the same explained in the section about direct cortical stimulation.

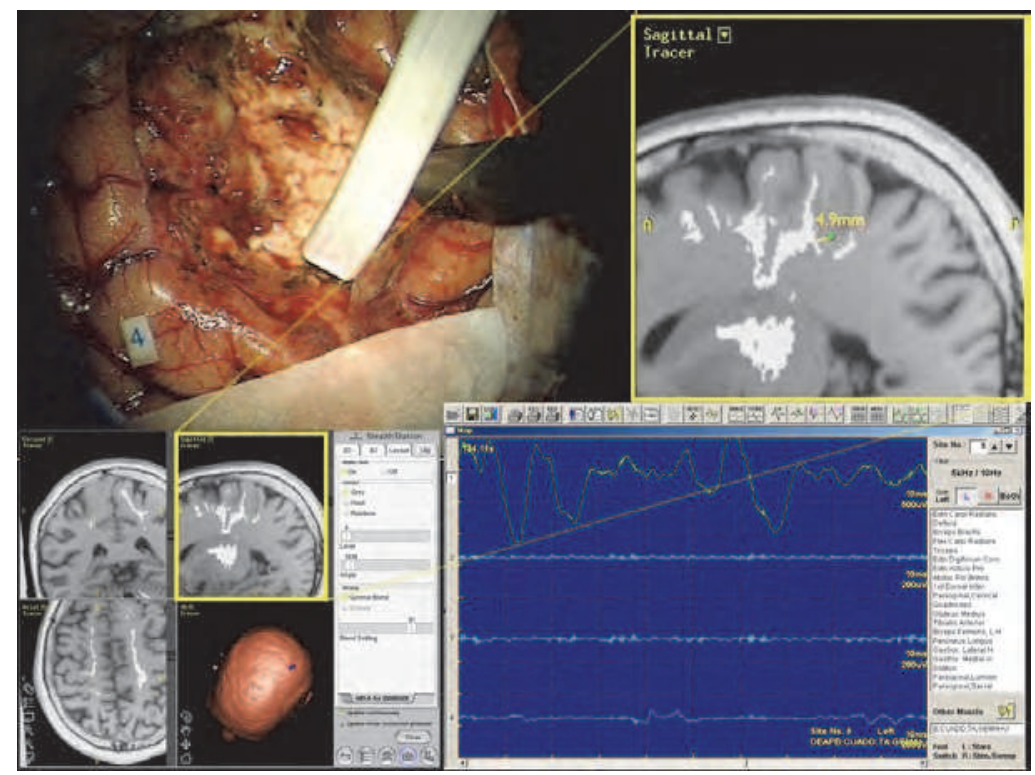

Fig. 17. Direct subcortical stimulation over the resection cavity walls surrounding fibers emerging from the primary motor area and its register 
Once removed the main tumor volume, the oncologic surgical concept forces to resect as much relative healthy tissue as possible, in order to complete a total macroscopic resection. This fact is easier when the tumor involves not functional fiber bundles, but in cases of highly eloquent white matter fiber tracts, the prize to pay by a total resection may be too high.

In this sense the direct subcortical stimulation appears as a great tool. The most accepted approach consists on stimulating the walls of the resection cavity each 3 or $4 \mathrm{~mm}$, using a bipolar stimulator during $2-4 \mathrm{sec}$, with intensities between 2 and $10 \mathrm{~mA}$ for the motor and sensitive pathways (Fig.17).

In cases of language areas, during tumor resection, the functional pathway mapping will follow outlining the eloquent cortical sites at depth. The patient should be nominating and counting in different phases until the end of tumor resection.

\section{Microsurgical treatment}

\subsection{Surgical approaches and patient's position}

Surgical position and approach is the result of a careful and concise preoperative surgical planning, and probably, one of the most important points to consider during the surgical procedure. Generally, it is assumed that a good position and surgical approach is performed when the venous return is facilitated, the patient's limbs are not forced, the surgeon has an optimal angle and vision of the field and all the neurosurgical team has space enough to develop their functions.

The preoperative imaging and functional tests can resemble very helpful to decide the surgical approach, however, the anatomic knowledge is basic to decide the patient's position in the operating room table. The patient's neck must not be forced to facilitate the venous return, so when necessary, different mechanisms will be used to elevate different parts of the body in order to get a free neck position. It is also a general recommendation to keep the head elevated between 6 and $10 \mathrm{~cm}$ from the level of the heart, fact that improves the venous return.

When the surgery is going to be performed with the patient awake, the position will be the same if possible, but the surgeon will employ all care with the aim that the patient is as comfortable as possible.

Patients undergoing craniotomies for intrinsic brain lesions are generally positioned in such a way that the lesion is highest in the field. However, the position and surgical approach in cases of tumors near eloquent areas will follow some general rules, depending on the location of the lesion (Fig.18).

\subsubsection{Precentral and postcentral areas at the sagittal level of the superior and middle frontal gyri. Anterior midline lesions in the cingulate gyrus}

In cases of tumors surrounding the paracentral lobe, it will be necessary to expose the rolandic cortex in order to facilitate stimulation and visual control of this area. The patient's head must be positioned straight up with a slightly flexion of the neck or $5-10^{\circ}$ turned to the contralateral side after fixation with the cephalostat device, in order to expose the field perpendicular to the surgeon's hands. The incision is usually performed in a horseshoe shape centered in the lesion and including the motor cortex. When the tumor remains located in the anterior cingulate gyrus or in the mesial side of the paracentral lobe, the incision must be highly extended to the contralateral side, in order to facilitate a crossing midline craniotomy. 

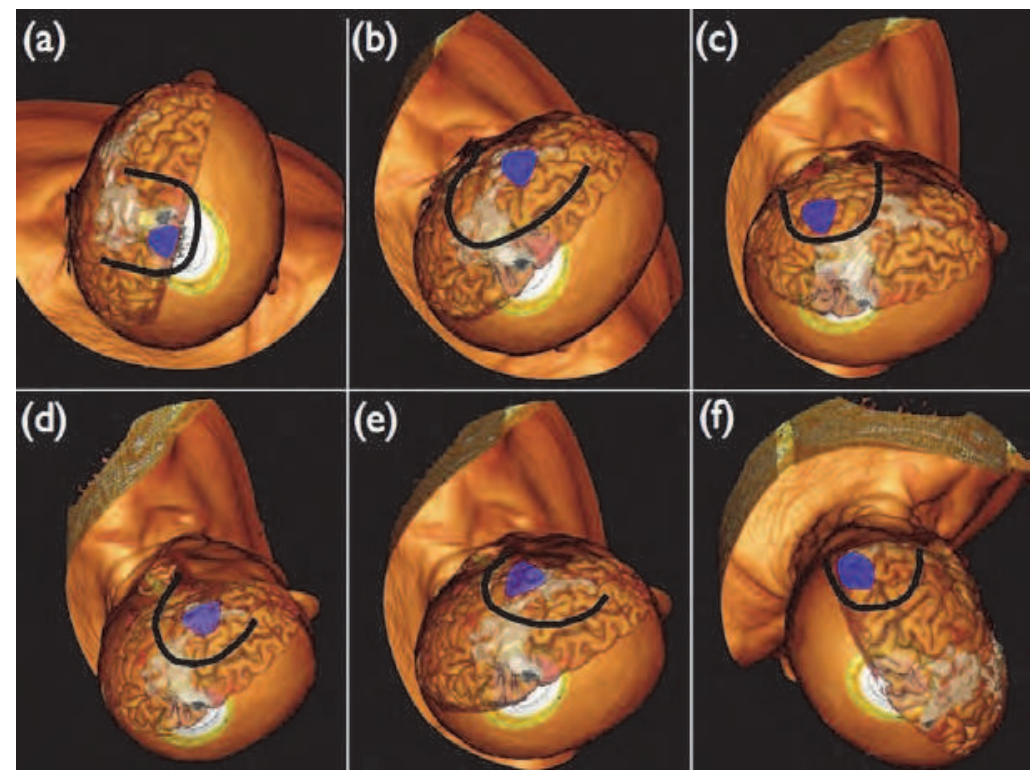

Fig. 18. Patient's position and skin incision for intrinsic brain tumors located in medial paracentral lobe (a), lateral paracentral lobe and Broca's area (b), angular and supramarginal gyrus and Wernicke's area (c), superior edge of insular lobe (d), inferior edge of insular lobe (e) and occipital lobe (f)

\subsubsection{Precentral and postcentral areas at the sagittal level of the inferior frontal gyrus. Pars triangularis and opercularis of the inferior frontal gyrus (Broca's Area)}

In these cases the approach philosophy is exactly the same than in more medial tumors of this area, so the rolandic cortex should be exposed, the tumor centered on the craniotomy and the field perpendicular to the surgeon's hand. To get these facts, positioning can be facilitated by turning the head between $45-60^{\circ}$ toward the contralateral side, elevating the ipsilateral shoulder to avoid forcing the ipsilateral side of the neck, improving with this maneuver the venous return. The skin incision can be performed in a horseshoe - shape not crossing the midline, or if preferred by the surgeon it can be traced as a $\mathrm{C}$ - shaped incision emerging above the zygomatic root, curving posteriorly enough to expose the lateral rolandic cortex and again curving anteriorly including the tumor till reaching the hair implantation line. This last skin incision is supposed to better respect the blood flow on the wound, making easier the healing process.

\subsubsection{Superior frontal gyrus (premotor area, supplementary motor area and frontal aye field)}

In cases of tumors affecting different parts of the superior frontal gyrus, the head position and skin incision is really similar to the midline rolandic lesions. The head is usually fixed in a central neutral position, not flexed and $8-10 \mathrm{~cm}$ elevated from the level of the heart. It is not necessary to expose the paracentral lobe, in fact it should be avoided, so the incision remains quite more anterior. The incision requires usually crossing the midline, so many times a posterior bicoronal incision replaces the typical horseshoe - shaped one. 


\subsubsection{Angular, supramarginal and posterior part of superior temporal gyri (Wernicke's area). Transverse gyrus of Heschl (primary auditive area)}

For approaching these tumors, the head is turned nearly $90^{\circ}$ to the contralateral side, elevating the shoulder as far as possible to get a free of tension neck, turning the head as far as getting it almost parallel to the floor. The incision is began at the root of zygoma, extending superiorly and then posteriorly to end well behind the pinna of the ear in a horseshoe - shaped (Berger \& Hadjipanayis, 2007), exactly the same as for a subtemporal approach. When the lesion affects the angular and supramarginal gyri, the incision can be moved quite more posterior and superior, even leaving the ear free. These incisions can be centered with the help of a neuronavigator system.

\subsubsection{Insular tumors}

Although the skin incision and craniotomy will always be the same (a extended pterional approach), the patient's position will highly depend on the relative location of the tumor inside the insula. The insula is hidden under the frontal, temporal and parietal opercula, so retraction is needed to approach this region. Avoiding retraction as much as possible is the reason to the different positions of the head, taking advantage from the force of gravity. When dealing with lesions affecting the superior aspect of insula (frontal part of the sulcus circularis insulae), the head will be turned $60-75^{\circ}$ to the contralateral side and extended at least $15^{\circ}$, to obtain a frontal lobe anatomical retraction when opening the sylvian fissure (Berger \& Hadjipanayis, 2007). In cases of tumors affecting the inferior part or temporal sulcus, and the limen insulae, the philosophy will be opposite, flexing the head $10-20^{\circ}$ in order to get a direct angle with minimal retraction.

\subsubsection{Lateral occipital lobe (primary visual area)}

In cases in which the tumor affects the occipital lobe the chosen position is the known as three - quarter prone position or park - bench. With this approach, the head is midly flexed and turned following the contralateral shoulder direction so that the nose points to the floor. This locates the occipital lobe in a nondependent fashion, avoiding pressure on the abdomen, although it is extremely difficult to be performed when dealing with obese patients. A sling supports the ipsilateral arm with a roll under the armpit to prevent brachial plexopathy, and the ipsilateral shoulder is taped to minimize its profile. A horseshoe shaped incision is then performed centered in the tumor.

\subsection{Surgical technique}

The development of neuroimaging, intraoperative functional mapping technologies and especially the incorporation of microneurosurgery skills to the intrinsic brain tumors surgery, has largely improved the extent of resection of these tumors with a great clinical impact over the postoperative morbidity and mortality.

\subsubsection{Transulcal microsurgical approach}

Understanding the gyral and sulcal anatomy is the first step in all neurosurgical procedures. Glial tumors grow in a completely different way from the rest of the human body neoplasms. They do not behave as a mass, but they present a special growing pattern, extending away the origin of the neoplasms using as corridors the vessels external walls and overall the white matter substance. In a given gyrus, there are at least two kinds of white 
matter fiber bundles, the arcuate or $U$ - shaped fibers that join adjacent gyri at the deep of the sulci, and long fiber bundles, which can be commissural or associative pathways. Modern neuroimaging has developed techniques, which offer a high definition about which gyri and white matter tracts are infiltrated, so the classical way to define a brain tumor location is nowadays dramatically changing. Thanks to these imaging modalities a brain tumor can be said to affect the pars opercularis of the inferior frontal gyrus and the frontal portion of the arcuate fascicle of the superior longitudinal fascicle in the left side, avoiding the classical nomenclature, which would have named it as a left frontal tumor.

All these information is needed to plan a surgical approach, and to reach a macroscopic complete resection of a given tumor. In this sense the surgical excision must be performed following the anatomical concepts offered by neuroimaging, so once localized the eloquent areas surrounding the tumor, and the affected gyri, the dissection will begin following the natural corridors of the brain, also known as sulci. Once completely deeply dissected the involved sulci and having respected the functional regions, a gross resection of the main tumor volume must be done.

The transulcal microsurgical resection remains of great interest, and brings the concept of oncologic resection to the field of brain tumors.

\subsubsection{Ultrasonic aspiration}

This technology was first introduced in the neurosurgical procedures 25 years ago, and actually has become an indispensable tool in the neurosurgical armamentarium for the resection of intracranial tumors.

The ultrasonic aspirator presents two effects over the tissue interface. The first effect supposes a suction that brings the surrounding tissue to the tip of the aspirator and forces it to vibrate, accelerate, and decelerate with the tip, fragmenting it away from harder tissues as vessels. The second important effect consists on a rapidly oscillating tip that produces localized pressure waves, which cause vapor pockets around cells in tissues with high water contents; the collapse of these pockets causes the tissue cells to rupture. The speed of fragmentation depends on the amplitude setting of the system (Jallo, 2001).

The use of this technology remains of great interest when dealing with intrinsic brain tumors, especially when these tumors are close to eloquent areas. Classical resection of intrinsic brain tumors is performed through an electric coagulating bipolar system to destroy tissue and a conventional suction system to aspirate it. This procedure can result as effective as ultrasonic aspiration, but there is a great difference. Bipolar coagulation systems increase the local temperature of the surrounding tissue and it has been demonstrated to be responsible of damaging neighbor areas to the resected tissue. On the contrary, the ultrasonic aspirator is at least as effective removing infiltrated tissue, but shows the advantage of not damaging the healthy surrounding healthy and sometimes functional tissue.

The use of ultrasonic aspirators can increase the extent of resection, respecting vessels and healthy surrounding tissue, and then improving the functional result.

\subsubsection{Subcortical resection}

A precise subcortical resection usually marks the difference between a good and an excellent brain tumor resection. Different procedures as intraoperative imaging modalities, neuronavigation systems, fluorescence - guidance and direct electrical stimulation, can help neurosurgeons on decision making about when to stop the tumor resection. 
Once performed the transulcal approach and the main tumor volume removal, the main challenge appears about when to stop the subcortical deep resection. This fact takes especial consideration in cases of adjacent white matter functional fiber bundles. To optimize this purpose the neurosurgeon's main armamentarium is based on different technical procedures.

Intraoperative imaging modalities as iMRI and intraoperative ultrasonography show in real - time the extent of resection, and in some cases they can offer the visualization of important fiber bundles near the walls of the resection cavity (Nimsky et al., 2005).

Other modalities as fluoresecence guided - resection, offer the ability of visualizing infiltrated tissue under a special wavelength, which could not be visualized under the usual white microscope light.

All these imaging and metabolic techniques are of great interest when resecting the tumoral mass, but in case of functional subcortical areas, the gold standard remains being the direct subcortical electric stimulation in order to localize the function and stop the resection to avoid postoperative morbidity. Different studies have been performed to study the optimal distance between the location of fiber tracts on the navigated DTI and the positive response to the subcortical electrical stimulation (Gonzalez-Darder et al., 2010; Nimsky et al., 2005), proposing a safety margin of $5-8 \mathrm{~mm}$. A positive response is accepted as to directly stimulate the involved fiber tract, when applying a stimulus of 2-8 mA during at least 2 seconds. The appearing of a positive response under these parameters must make the surgeon to stop the resection in that area.

\section{Conclusions}

The integration of pre- and intraoperative anatomical, imaging and functional studies allows for a functional resection that significantly widens the extent of resection in lesions in relevant eloquent areas. Navigation allows neurosurgeons to integrate and understand the correlation between the preoperative data and the intraoperative findings. The cortical functional areas are anatomically and functionally defined in the preoperative period by using MR and fMR imaging studies, as well as other non-invasive techniques as the Transcranial Magnetic Stimulation, and the subcortical functional areas are defined by DTI based tractography, whereas intraoperative confirmation is achieved using CS and the N20 wave inversion study for the cortical areas and sCS for the subcortical areas. Microsurgical treatment supported by intraoperative imaging techniques and fluorescence, guided by navigation and with the aid of the studies described above, allows great tumor resections in lesions located in cortical and subcortical eloquent motor areas, with a relatively high neurological morbidity in the immediate postoperative period, which is significantly reduced both quantitatively and qualitatively during the first postoperative weeks. The ongoing studies should define safety margins for functional resection that take the brain shift into consideration. Finally, the benefit of these protocols for disease-free interval, recurrence, or ultimate patient survival remains to be defined in future.

\section{Acknowledgements}

The authors would like to thank the collaboration of all medical and nonmedical members of the Services Neurosurgery, Neurophysiology and Anesthesiology from Hospital Clinico Universitario, Valencia; of ERESA (Exploraciones Radiológicas SA), and of the Department of Basic Psychology, and Clinical Psychobiology from UJI (Universitat Jaume I). Also thank 
to Medtronic by their technical support. This work was supported in part by the grant 2008/005 Foundation ERESA Group for Development and Research Medical Area (Neurology).

\section{References}

Basser P.J., Pajevic S., Pierpaoli C., Duda J. \& Aldroubi A. (2000). In vivo fiber tractography using DT-MRI data. Magnetic Resonance Medicine, Vol.44, pp625-632

Berger M.S., Deliganis A.V., Dobbins J. \& Keles G.E. (1994). The effect of extent of resection on recurrence in patients with low grade cerebral hemisphere gliomas. Cancer, Vol.74, pp1784-1791

Berger M.S. \& Hadjipanayis C.G. (2007). Surgery of intrinsic cerebral tumors. Neurosurgery, Vol.61: pp279-305

Brandes A.A., Scelzi E., Salmistraro G., Ermani M., Carollo C., Berti F., Zampieri P., Baiocchi C. \& Fiorentino M.V. (1997). Incidence of risk of thromboembolism during treatment of high - grade gliomas: a prospective study. European Journal of Cancer, Vol.33, pp-1592-1596

Cha S. (2006). Update on brain tumor imaging: from anatomy to physiology. American Journal of Neuroradiology, Vol.27, pp475-487

Claes A., Idema A.J. \& Wesseling P. (2007). Diffuse glioma growth: a guerilla war. Acta Neuropathologica, Vol.114, pp443-458

Forster M.T., Hattingen E., Senft C., Gasser T., Seifert V. \& Szelényi A. (2011). Navigated Transcranial Magnetic Stimulation and functional Magnetic Resonance Imaging advanced adjuncts in preoperative planning for central region tumors. Neurosurgery, 2011 Jan 26. [Epub ahead of print]

French J.A., Kanner A.M., Bautista J., Abou-Khalil B., Browne T., Harden C.L., Theodore W.H., Bazil C., Stern J., Schachter S.C., Bergen D., Hirtz D., Montouris G.D., Nespeca M., Gidal B., Marks W.J., Turk W.R., Fischer J.H., Bourgeois B., Wilner A., Faught R.E., Sachdeo R.C., Beydoun A. \& Glauser T.A. (2004). Efficacy and tolerability of the new antiepileptic drugs I: treatment of new onset epilepsy: report of the therapeutics and technology assessment subcommittee of the American Academy of Neurology and the American Epilepsy Society. Neurology, Vol.62, pp1252-1260

Gallucci M., Capoccia S. \& Catalucci A. (2007). Functional Systems, In: Radiographic atlas of skull and brain anatomy, Heilmann U., Stoeck M. \& Steinen-Bro F. (ed.), pp208-262, Springer-Verlag, ISBN-10 3-540-34190-0, New York.

Giese A., Loo M.A., Rief M.D., Tran N. \& Berens M.E. (1995). Substrates for astrocytoma invasion. Neurosurgery, Vol.37, pp294-302

Giese A., Kluwe L., Laube B., Meissner H., Berens M.E. \& Westphal M. (1996). Migration of human glioma cells on myelin. Neurosurgery, Vol.38, pp755-764

Giese A., Bjerkvig R., Berens M.E. \& Westphal M. (2003). Cost of migration: invasion of malignant gliomas and implications for treatment. Journal of Clinical Oncology, Vol.21, pp1624-1636

Glantz M.J., Cole B.F., Forsyth PA, Recht L.D., Wen P.Y., Chamberlain M.C., Grossman S.A. \& Cairncross J.G. (2000). Practice parameter: anticonvulsant prophylaxis in patients with newly diagnosed brain tumors. Report of the quality standards subcommittee of the American Academy of Neurology. Neurology, Vol.54, pp1886-1893

González-Darder J.M., González-López P., Talamantes F., Quilis V., Cortés V., García-March G. \& Roldán P. (2010). Multimodal navigation in the functional microsurgical 
resection of intrinsic brain tumors located in eloquent motor areas: role of tractography. Neurosurgical Focus, Vol.28(2), ppE5

Hamilton M.G., Yee W.H., Hull R.D. \& Ghali W.A. (2011). Venous thromboembolism prophylaxis in patients undergoing cranial neurosurgery: a systematic review and meta - analysis. Neurosurgery, Vol.68, pp571-581

Hwang J.H., Smith C.A., Salhia B. \& Rutka J.T. (2008). The role of fascin in the migration and invasiveness of malignant glioma cells. Neoplasia, Vol.10, pp149-159

Jallo G.I. (2001). CUSA EXcel Ultrasonic Aspiration System. Neurosurgery Vol.48, pp695-697

Kaal E.C. \& Vecht C.J. (2004). The management of brain edema in brain tumors. Current Opinion in Oncology, Vol.16(6), 593-600

Keles G.E., Lamborn K.R. \& Berger M.S. (2001). Low - grade hemispheric gliomas in adults: a critical review of extent of resection as a factor influencing outcome. Journal of Neurosurgery, Vol.95, pp735-745

Keles G.E., Chang E.F., Lamborn K.R., Tihan T., Chang C.J., Chang S.M. \& Berger M.S. (2006). Volumetric extent of resection and residual contrast enhancement on initial surgery as predictors of outcome in adult patients with hemispheric anaplastic astrocytoma. Journal of Neurosurgery, Vol.105, pp34-40

Kim P.E. \& Zee C.S. (2007). Imaging of the cerebrum. Neurosurgery, Vol.61, ppSHC-123SHC-146

Maranhao E.T., Maranhao-Filho P., Lima M.A. \& Vincent M.B. (2010). Can clinical tests detect early signs of monohemispheric brain tumors? Journal of Neurologic Physical Therapy, Vol.34, pp145-149

Marras L.C., Geerts W.H., \& Perry J.R. (2000). The risk of venous thromboembolism is increased throughout the course of malignant glioma: an evidence - based review. Cancer, Vol.89, pp640-646

McGirt M.J., Chaichana K.L., Attenello F.J., Weingart J.D., Than K., Burger P.C., Olivi A., Brem H. \& Quinoñes-Hinojosa A.R. (2008). Extent of surgical resection is independently associated with survival in patients with hemispheric infiltrating low-grade gliomas. Neurosurgery, Vol.63, pp700-708

McGirt M.J., Chaichana K.L., Gathinji M., Attenello F.J., Than K., Olivi A., Weingart J.D., Brem H. \& Quinoñes-Hinojosa A.R. (2009). Independent association of extent of resection with survival in patients with malignant brain astrocytoma. Journal of Neurosurgery, Vol.110, pp156-162

Moots P.L., Maciunas R.J., Eisert D.R., Parker R.A., Laporte K. \& Abou-Khalil B. (1995). The course of seizure disorders in patients with malignant gliomas. Archives of Neurology, Vol.52, pp717-724

Nimsky C., Ganslandt O., Hastreiter P., Wang R., Benner T., Sorensen A.G. \& Fahlbusch R. (2005). Preoperative and intraoperative diffusion tensor imaging based fiber tracking in glioma surgery. Neurosurgery, Vol.56, pp130 -137

North J.B., Penhall R.K., Hanieh A., Frewin D.B. \& Taylor W.B. (1983). Phenytoin and postoperative epilepsy. Journal of Neurosurgery Vol.58, pp672-677

Ostergaard L. (2005): Principles of cerebral perfusion imaging by bolus tracking. Journal of Magnetic Resonance Imaging, Vol.22, pp710-717

Papadopoulos M.C., Saadoun S., Davies D.C. \& Bell B.A.: Emerging molecular mechanisms of brain tumor oedema. British Journal of Neurosurgery, Vol.15, pp101-108

Pesudo J.V., González-Darder J.M., Feliu R., Belloch V., Vera J. \& Gil J.L. (2001). Assessment of the degree of resection of high grade supratentorial gliomas with early postoperative magnetic resonance. Neurocirugia Vol.12, pp43-50 
Pouratian N., Cannestra A.F., Bookheimer S.Y., Martin N.A. \& Toga A.W. (2004). Variability of intraoperative electrocortical stimulation mapping parameters across and whithin individuals. Journal of Neurosurgery, Vol.101, pp458-466

Price S.J., Jena R., Burnet N.G., Hutchinson P.J., Dean A.F., Peña A., Pickard J.D., Carpenter T.A \& Gillard J.H. (2006). Improved delineation of glioma margins and regions of infiltration with the use of diffusion tensor imaging: an image-guided biopsy study. American Journal of Neuroradiology, Vol.27, pp1969-1974

Provenzale J.M., McGraw P., Mhatre P., Guo A.C. \& Delong D. (2004). Peritumoral brain regions in gliomas and meningiomas: investigation with isotropic diffusion-weighted MR imaging and diffusion-tensor MR imaging. Radiology, Vol.232, pp451-460

Ribas G.C. (2010). The cerebral sulci and gyri. Neurosurgical Focus, Vol.28(2), ppE2

Rosenfeld M.R. \& Pruitt A.A. (2007). Managing medical complications in patients with brain tumors. Community Oncology, Vol.4, pp411-416

Ruohonen J. \& Karhu J. (2010). Navigated transcranial magnetic stimulation. La stimulation magnétique transcrânienne. Clinical Neurophysiology, Vol.40, pp7-17

Sanai N. \& Berger M.S. (2008). Glioma extent of resection and its impact on patient outcome. Neurosurgery, Vol.62, pp753-766

Sawaya R., Hammoud M., Schoppa D., Hess K.R., Wu S.Z., Shi W.M. \& Wildrick D.M. (1998). Neurosurgical outcomes in a modern series of 400 craniotomies for treatment of parenchymal tumors. Neurosurgery, Vol.42(5), pp1044-1055

Schaller B. \& Ruegg S.J. (2003). Brain tumor and seizures: pathophysiology and its implications for treatment revisited. Epilepsia, Vol.44(9), pp1223-1232

Schulder M. \& Carmel P.W. (2003). Intraoperative magnetic resonance imaging: impact on brain tumor surgery. Cancer Control Journal, Vol.10, pp115-124

Shaw E.G., Berkey B., Coons S.W., Bullard D., Brachman D., Buckner J.C., Stelzer K.J., Barger G.R., Brown P.D., Gilbert M.R. \& Mehta M. (2008). Recurrence following neurosurgeondetermined gross-total resection of adult supratentorial low-grade glioma: results of a prospective clinical trial. Journal of Neurosurgery, Vol.109, pp835-841

Stummer W., Stocker S., Wagner S., Stepp H., Fritsch C., Goetz C., Goetz A.E., Kiefmann R. \& Reulen H.J. (1998). Intraoperative detection of malignant gliomas by 5 -aminolevulinic acid-induced porphyrin fluorescence. Neurosurgery, Vol.42, pp518-526

Stummer W., Pichlmeier U., Meinel T., Wiestler O.D., Zanella F. \& Reulen H.J. (2006). Fluorescence-guided surgery with 5-aminolevulinic acid for resection of malignant glioma: a randomised controlled multicentre phase III trial. The Lancet Oncology, Vol.7(5), pp392-401

Tanaka Y., Nariai T., Momose T., Aoyagi M., Maehara T., Tomori T., Yoshino Y., Nagaoka T., Ishiwata K., Ishii K. \& Ohno K. (2009). Glioma surgery using a multimodal navigation system with integrated metabolic images. Journal of Neurosurgery, Vol.110, pp163-172

Tharin S. \& Golby A. (2007). Functional brain mapping and its applications to neurosurgery. Neurosurgery, Vol.60, ppONS-185-ONS-202

Vahala E., Ylihautala M., Tuominen J., Schiffbauer H., Katisko J., Yrjänä S., Vaara T., Ehnholm G. \& Koivukangas J. (2001). Registration in interventional procedures with optical navigator. Journal of Magnetic Resonance Imaging, Vol.13(1), pp93-98

Vick N.A. \& Wilson C.B. (1985). Total care of the patient with a brain tumor. Neurologic Clinics, Vol.3, pp705-710

Wen P.Y., Schiff D., Kesari S., Drappatz J., Gigas D.C. \& Doherty L. (2006). Medical management of patients with brain tumors. Journal of Neurooncology, Vol.80, pp313332 


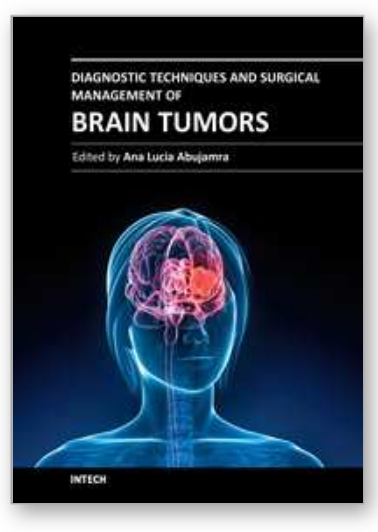

\author{
Diagnostic Techniques and Surgical Management of Brain Tumors \\ Edited by Dr. Ana Lucia Abujamra
}

ISBN 978-953-307-589-1

Hard cover, 544 pages

Publisher InTech

Published online 22, September, 2011

Published in print edition September, 2011

The focus of the book Diagnostic Techniques and Surgical Management of Brain Tumors is on describing the established and newly-arising techniques to diagnose central nervous system tumors, with a special focus on neuroimaging, followed by a discussion on the neurosurgical guidelines and techniques to manage and treat this disease. Each chapter in the Diagnostic Techniques and Surgical Management of Brain Tumors is authored by international experts with extensive experience in the areas covered.

\title{
How to reference
}

In order to correctly reference this scholarly work, feel free to copy and paste the following:

José Manuel González - Darder and Pablo González - López (2011). Management of Brain Tumors in Eloquent Areas, Diagnostic Techniques and Surgical Management of Brain Tumors, Dr. Ana Lucia Abujamra (Ed.), ISBN: 978-953-307-589-1, InTech, Available from: http://www.intechopen.com/books/diagnostictechniques-and-surgical-management-of-brain-tumors/management-of-brain-tumors-in-eloquent-areas

\section{INTECH}

open science | open minds

\section{InTech Europe}

University Campus STeP Ri

Slavka Krautzeka 83/A

51000 Rijeka, Croatia

Phone: +385 (51) 770447

Fax: +385 (51) 686166

www.intechopen.com

\section{InTech China}

Unit 405, Office Block, Hotel Equatorial Shanghai

No.65, Yan An Road (West), Shanghai, 200040, China

中国上海市延安西路65号上海国际贵都大饭店办公楼 405 单元

Phone: +86-21-62489820

Fax: $+86-21-62489821$ 
(C) 2011 The Author(s). Licensee IntechOpen. This chapter is distributed under the terms of the Creative Commons Attribution-NonCommercialShareAlike-3.0 License, which permits use, distribution and reproduction for non-commercial purposes, provided the original is properly cited and derivative works building on this content are distributed under the same license. 